\title{
Swiss-cheese action on the totalization of action-operads
}

\author{
JULIEN DUCOULOMBIER
}

\begin{abstract}
We prove that, if a pair of semicosimplicial spaces $\left(X_{c}^{\bullet} ; X_{o}^{\bullet}\right)$ arises from a colored operad, then the semitotalization $\operatorname{sTot}\left(X_{o}^{\bullet}\right)$ has the homotopy type of a relative double loop space and the pair $\left(\operatorname{sTot}\left(X_{c}^{\bullet}\right) ; \operatorname{sTot}\left(X_{o}^{\bullet}\right)\right)$ is weakly equivalent to an explicit algebra over the two dimensional Swiss-cheese operad $\mathcal{S C}_{2}$.
\end{abstract}

$55 \mathrm{P} 48,55 \mathrm{P} 47$

\section{Introduction}

A multiplicative operad $O$ is an operad under the associative operad As. In [15], McClure and Smith build a cosimplicial space $O^{\bullet}$ from the multiplicative operad $O$ and show that, under some conditions, its homotopy totalization is a double loop space. V Turchin in [19] and, independently, Dwyer and Hess in [7] are able to identify the space of double delooping and prove, under the assumption $O(0) \simeq O(1) \simeq *$, that

$$
\operatorname{hoTot}\left(O^{\bullet}\right) \simeq \Omega^{2} \operatorname{Operad}^{h}(\text { As; } O),
$$

where $\operatorname{Operad}^{h}(\mathrm{As} ; O)$ is the space of derived maps from the associative operad to $O$. In order to prove this statement, Turchin introduces the categories of bimodules and infinitesimal bimodules over an operad $O$, denoted respectively by $\operatorname{Bimod}_{O}$ and $\operatorname{Ibimod}_{O}, \operatorname{such}$ that $\operatorname{hoTot}\left(O^{\bullet}\right)$ is weakly equivalent to $\operatorname{Ibimod}_{\mathrm{As}}^{h}(\mathrm{As} ; O)$. Then he proves the following two weak equivalences:

$$
\begin{aligned}
\operatorname{Ibimod}_{\mathrm{As}}^{h}(\mathrm{As} ; O) & \simeq \Omega \operatorname{Bimod}_{\mathrm{As}}^{h}(\operatorname{As} ; O), \\
\operatorname{Bimod}_{\mathrm{As}}^{h}(\mathrm{As} ; O) & \simeq \Omega \operatorname{Operad}^{h}(\mathrm{As} ; O) .
\end{aligned}
$$

This result was motivated by the following theorem of D Sinha: the space of long knots $\operatorname{Emb}_{c}\left(\mathbb{R} ; \mathbb{R}^{d}\right)$ has the homotopy type of $\operatorname{hoTot}\left(\mathcal{K}_{d}^{\bullet}\right)$, where $\mathcal{K}_{d}$ is a multiplicative operad weakly equivalent to the little $d$-disk operad. The Swiss-cheese operad $\mathcal{S C}_{d}$ is a relative version of the little disc operad. It is a two-colored topological operad with set of colors $S=\{o ; c\}$ that has been introduced by A Voronov in [21]. In particular, if $f: A \rightarrow X$ is a pointed continuous map, then the following pair is an $\mathcal{S C}_{d}$-space:

$$
\left(\Omega^{d} X ; \Omega^{d}(X ; A)\right):=\left(\Omega^{d} X ; \operatorname{hofib}\left(\Omega^{d-1} A \rightarrow \Omega^{d-1} X\right)\right) .
$$


In this paper we make great use of the operad $\pi_{0}\left(\mathcal{S C}_{1}\right)$, which is the operad of monoid actions $\mathcal{A}$ ct: it is a $2-$ colored operad whose algebras are the pairs of spaces $(X ; A)$, where $X$ is a monoid and $A$ a left $X$-module. The operad $\mathcal{A c t}>0$ is the nonunital version of $\mathcal{A} c t$. Similarly to the uncolored case, there is a notion of an $\mathcal{A c t}_{>0}$-bimodule and an $\mathcal{A c t}_{>0}$-infinitesimal bimodule. We prove that, if $O$ is an operad under $\mathcal{A} c t$, then it gives rise to a pair of semicosimplicial spaces $\left(O_{c} ; O_{o}\right)$ such that the pair $\left(\operatorname{sTot}\left(O_{c}\right) ; \operatorname{sTot}\left(O_{o}\right)\right)$ is weakly equivalent to

$$
\left(\Omega^{2} \operatorname{Operad}^{h}\left(\operatorname{As}_{>0} ; O_{c}\right) ; \Omega^{2}\left(\operatorname{Operad}^{h}\left(\operatorname{As}_{>0} ; O_{c}\right) ; \operatorname{Operad}^{h}\left(\mathcal{A c t}_{>0} ; O\right)\right)\right) ;
$$

that is, it is an $\mathcal{S C}_{2}$-space.

Organization of the paper The paper is divided into six sections. Section 1 is an introduction. It describes the categories of colored operads, bimodules and infinitesimal bimodules over an operad. An explicit description of a point $X$ in $\operatorname{Bimod}_{\mathcal{A c t}>0}$ and $\operatorname{Ibimod}_{\mathcal{A} t_{>0}}$ in terms of pairs of semicosimplicial spaces $\left(X_{c} ; X_{o}\right)$ is given. We insist on the link between bimodule structures over $\mathcal{A} c t_{>0}$ and monoidal structures on semicosimplicial spaces introduced by McClure and Smith in [15].

Section 2 introduces the left adjoint functors to the forgetful functors from the categories of bimodules and infinitesimal bimodules over an $S$-colored operad to the category of $S$-sequences. These adjunctions will be used in Section 3 in order to define a model category structure on $\operatorname{Bimod}_{O}$ and $\operatorname{Ibimod}_{O}$. We also determine an explicit cofibrant replacement of $\mathcal{A} c t$ (resp. $\mathcal{A c t}_{>0}$ ) in the model category $\operatorname{Ibimod}_{\mathcal{A} c t>0}$ (resp. $\left.\operatorname{Bimod}_{\mathcal{A c t}>0}\right)$ and prove the weak equivalence

$$
\operatorname{Ibimod}_{\mathcal{A} t>0}^{h}(\mathcal{A c t} ; M) \simeq \operatorname{Ibimod}_{\mathrm{As}>0}^{h}\left(\mathrm{As} ; M_{c}\right),
$$

where $M$ is an $\mathcal{A} c t_{>0}$-infinitesimal bimodule and $M_{c}$ is its closed part.

In Section 4 we prove the first relative delooping theorem. From an $\mathcal{A c t}_{>0}$-bimodule map $\eta: \mathcal{A} t \rightarrow M$, we extract two semicosimplicial spaces $\left(M_{c} ; M_{o}\right)$. We prove, under some conditions, the following weak equivalence of pairs:

$\left(\operatorname{sTot}\left(M_{c}\right) ; \operatorname{sTot}\left(M_{o}\right)\right) \simeq$

$\left(\Omega \operatorname{Bimod}_{\mathrm{As}_{>0}}^{h}\left(\operatorname{As}_{>0} ; M_{c}\right) ; \Omega\left(\operatorname{Bimod}_{\mathrm{As}_{>0}}^{h}\left(\operatorname{As}_{>0} ; M_{c}\right) ; \operatorname{Bimod}_{\mathcal{A c t}>0}^{h}\left(\mathcal{A c t}_{>0} ; M\right)\right)\right)$.

Section 5 consists in considering a particular case where a double relative delooping theorem holds. Namely let $\alpha$ : As $\rightarrow O$ be a map of operads and $\beta: O \rightarrow B$ be a map of $O$-bimodules. The two objects $O$ and $B$ are equipped with semicosimplicial structures. Under some conditions, we prove the weak equivalence of pairs

$(\operatorname{sTot}(O) ; \operatorname{sTot}(B)) \simeq$

$\left(\Omega^{2} \operatorname{Operad}^{h}\left(\operatorname{As}_{>0} ; O\right) ; \Omega^{2}\left(\operatorname{Operad}^{h}\left(\operatorname{As}_{>0} ; O\right) ; \operatorname{Operad}_{\{o ; c\}}^{h}\left(\mathcal{A c t}_{>0} ; X\right)\right)\right)$, 
where $X$ is a colored operad build out of $O$ and $B$.

Section 6 is devoted to the proof of the main theorem: if $O$ is an $\{o ; c\}$-operad under Act such that $O(0 ; c) \simeq O(1 ; c) \simeq O(1 ; o) \simeq *$, then we have the weak equivalence of pairs

$\left(\operatorname{sTot}\left(O_{c}\right) ; \operatorname{sTot}\left(O_{o}\right)\right) \simeq$

$$
\left(\Omega^{2} \operatorname{Operad}^{h}\left(\operatorname{As}_{>0} ; O_{c}\right) ; \Omega^{2}\left(\operatorname{Operad}^{h}\left(\operatorname{As}_{>0} ; O_{c}\right) ; \operatorname{Operad}_{\{o ; c\}}^{h}\left(\mathcal{A c t}_{>0} ; O\right)\right)\right) .
$$

Convention By space we mean compactly generated Hausdorff space, and by abuse of notation we denote by Top this category; see eg Section 2.4 in Hovey [14]. If $X$, $Y$ and $Z$ are spaces, then $\operatorname{Top}(X ; Y)$ is equipped with the compact-open topology in order to have a homeomorphism $\operatorname{Top}(X ; \operatorname{Top}(Y ; Z)) \cong \operatorname{Top}(X \times Y ; Z)$.

A semicosimplicial space $X^{\bullet}$ is a family of topological spaces $\left\{X^{n} \mid n \geq 0\right\}$ endowed with operations

$$
d^{i}: X^{n} \rightarrow X^{n+1} \quad \text { for } i \in\{0, \ldots, n+1\},
$$

satisfying the cosimplicial relations $d^{j} d^{i}=d^{i} d^{j-1}$ for $0 \leq i<j$. By semitotalization $\operatorname{sTot}\left(X^{\bullet}\right)$ we mean the space of natural transformations from the semicosimplicial space $\Delta^{\bullet}$ to $X^{\bullet}$. From a cosimplicial space, the semitotalization of the underlying semicosimplicial space is also called the fat-totalization, which is a homotopy invariant. Since the homotopy totalization of a cosimplicial space is weakly equivalent to the semitotalization (see Lemma 3.8 in Dror and Dwyer [6]), we will ignore the codegeneracies in the present work. We denote weak equivalences by the symbol $\simeq$.

\section{Bimodules and infinitesimal bimodules over a colored operad}

In what follows we introduce the category of colored operads as well as the categories of bimodules and infinitesimal bimodules over a colored operad. We focus on the operads with two colors $\{o ; c\}$, called $\{o ; c\}$-operads. In particular, we define the $\{o ; c\}$-operad $\mathcal{A c t}_{>0}$ of monoid actions as in [13]. Besides, we characterize the bimodules and infinitesimal bimodules over this operad in terms of semicosimplicial spaces.

\section{A The operad of (unital) monoid actions}

Definition 1.1 Let $S$ be a set. An $S$-sequence is a collection of topological spaces $\left\{O\left(s_{1}, \ldots, s_{n} ; s_{n+1}\right)\right\}_{s_{i} \in S}^{n \in \mathbb{N}}$. The set $S$ is called the set of colors. A map between two $S$-sequences $O_{1}$ and $O_{2}$ is a collection of continuous maps

$$
\left\{f_{s_{1}, \ldots, s_{n} ; s_{n+1}}: O_{1}\left(s_{1}, \ldots, s_{n} ; s_{n+1}\right) \rightarrow O_{2}\left(s_{1}, \ldots, s_{n} ; s_{n+1}\right)\right\}_{s_{i} \in S}^{n \in \mathbb{N}} .
$$


We denote by $\operatorname{Coll}(S)$ the category of $S$-sequences.

Notation 1.2 If $M$ is an $\{o ; c\}$-sequence, then we use the following notation in the rest of the text:

$$
M_{c}^{n}=M(n ; c)=M(\underbrace{c, \ldots, c}_{n} ; c) \quad \text { and } \quad M_{o}^{n}=M(n+1 ; o)=M(\underbrace{c, \ldots, c}_{n}, o ; o) .
$$

We denote by $M_{c}$ the family $\left\{M_{c}^{n}\right\}_{n \geq 0}$ and by $M_{o}$ the family $\left\{M_{o}^{n}\right\}_{n \geq 0}$.

Definition 1.3 An $S$-operad is an $S$-sequence $O$ endowed with operations $\circ_{i}: O\left(s_{1}, \ldots, s_{n} ; s_{n+1}\right) \times O\left(s_{1}^{\prime}, \ldots, s_{m}^{\prime} ; s_{i}\right)$

$$
\rightarrow O\left(s_{1}, \ldots, s_{i-1}, s_{1}^{\prime}, \ldots, s_{m}^{\prime}, s_{i+1}, \ldots, s_{n} ; s_{n+1}\right),
$$

for $1 \leq i \leq n$, and distinguished elements $\left\{*_{s} \in O(s ; s)\right\}_{s \in S}$ satisfying associativity and unit axioms [1]. We denote by $x \circ_{i} y$ the operation $\circ_{i}(x ; y)$ for $x, y \in O$. Define Operad $_{S}$ to be the category of $S$-operads, where a map of $S$-operads is an $S$-sequence map that preserves the operadic structure.

Let $O$ be an $S$-operad and $A=\left\{A_{S}\right\}_{s \in S}$ be a family of topological spaces. The endomorphism $S$-operad End $_{A}$ (see [4]) is the family of spaces of continuous maps defined by

$$
\operatorname{End}_{A}\left(s_{1}, \ldots, s_{n} ; s_{n+1}\right)=\operatorname{Top}\left(A_{s_{1}} \times \cdots \times A_{s_{n}} ; A_{s_{n+1}}\right) .
$$

The family $A$ is called an $O$-space if there exists a map of $S$-operads $O \rightarrow \operatorname{End}_{A}$.

Definition 1.4 [13] Let $S=\{o, c\}$. The $S$-operad of monoid actions $\mathcal{A}_{>0}$ is given by the $S$-sequence

$$
\mathcal{A c t}_{>0}(n ; c)=*_{n ; c} \quad \text { for } n>0, \quad \mathcal{A c t}_{>0}(n ; o)=*_{n+1 ; o} \text { for } n>0,
$$

and the empty set otherwise, with $*_{n ; c}$ and $*_{n ; o}$ each being the one-point topological space. The compositions are as follows:

$$
\left\{\begin{array}{l}
*_{n ; c} \circ_{i} *_{m ; c}=*_{n+m-1 ; c}, \\
*_{n ; o} \circ_{i} *_{m ; c}=*_{n+m-1 ; o} \\
*_{n ; o} \circ_{n} *_{m ; o}=*_{n+m-1 ; o},
\end{array} \text { for } i \neq n,\right.
$$

Similarly, the $S$-operad of unital monoid actions $\mathcal{A c t}$ is given by the $S$-sequence

$$
\mathcal{A c t}_{>0}(n ; c)=*_{n ; c} \quad \text { for } n \geq 0, \quad \mathcal{A c t}_{>0}(n ; o)=*_{n ; o} \quad \text { for } n>0,
$$

and the empty set otherwise, with the same compositions. Consequently, the $S$-operad $\mathcal{A} c t$ (resp. $\left.\mathcal{A} c t_{>0}\right)$ is generated by $*_{0 ; c}, *_{2 ; c}$ and $*_{2 ; o}\left(\right.$ resp. $*_{2 ; c}$ and $\left.*_{2 ; o}\right)$. 
An $\mathcal{A}$ ct-space is a pair of topological spaces $(X ; A)$ with $X$ a topological monoid with unit and $A$ a left module over $X$.

The $\{c\}$-sequence given by the restriction of $\mathcal{A} c t$ (resp. $\left.\mathcal{A} c t_{>0}\right)$ to the color $\{c\}$ is the associative operad As (resp. the strict associative operad $\mathrm{As}_{>0}$ ). We use the notation $*_{n}$ to refer to the one-point topological space $\operatorname{As}(n)$.

The operad of monoid actions has been introduced by Hoefel, Livernet and Stasheff [13] in the context of the recognition principle for relative loop spaces.

Definition 1.5 We define an action-operad to be an $\{o ; c\}$-operad $O$ equipped with a map $\eta: \mathcal{A c t} \rightarrow O$.

\section{B Infinitesimal bimodules over a colored operad}

Definition 1.6 Let $O$ be an $S$-operad. An infinitesimal bimodule over the operad $O$ (or $O$-infinitesimal bimodule) is an $S$-sequence $M$ endowed with operations

$$
\begin{aligned}
& \circ_{i}: O\left(s_{1}, \ldots, s_{n} ; s_{n+1}\right) \times M\left(s_{1}^{\prime}, \ldots, s_{m}^{\prime} ; s_{i}\right) \\
& \rightarrow M\left(s_{1}, \ldots, s_{i-1}, s_{1}^{\prime}, \ldots, s_{m}^{\prime}, s_{i+1}, \ldots, s_{n} ; s_{n+1}\right), \\
& \mathrm{o}^{i}: M\left(s_{1}, \ldots, s_{n} ; s_{n+1}\right) \times O\left(s_{1}^{\prime}, \ldots, s_{m}^{\prime} ; s_{i}\right) \\
& \rightarrow M\left(s_{1}, \ldots, s_{i-1}, s_{1}^{\prime}, \ldots, s_{m}^{\prime}, s_{i+1}, \ldots, s_{n} ; s_{n+1}\right),
\end{aligned}
$$

for $1 \leq i \leq n$, satisfying associativity and unit relations [1]. A map between $O-$ infinitesimal bimodules is given by an $S$-sequence map preserving this structure. Let Ibimod $O_{O}$ be the category of infinitesimal bimodules over $O$. We denote by $x o_{i} y$ (resp. $x \circ^{i} y$ ) the operation $\circ_{i}(x ; y)$ (resp. $\left.\circ^{i}(x ; y)\right)$ with $x \in O$ and $y \in M$ (resp. $x \in M$ and $y \in O)$.

Example 1.7 If $\eta: O_{1} \rightarrow O_{2}$ is any $S$-operad map, then $O_{2}$ is endowed with the following $O_{1}$-infinitesimal bimodule structure:

$$
\circ_{i}: O_{1} \times O_{2} \stackrel{\eta \times \mathrm{id}}{\longrightarrow} O_{2} \times O_{2} \stackrel{\circ_{i}}{\longrightarrow} O_{2}
$$

and

$$
\circ^{i}: O_{2} \times O_{1} \stackrel{\mathrm{id} \times \eta}{\longrightarrow} O_{2} \times O_{2} \stackrel{{ }^{\circ}}{\longrightarrow} O_{2}
$$

Consequently, if $A$ is an $O$-space, then $\operatorname{End}_{A}$ is an $O$-infinitesimal bimodule.

Definition 1.8 Let $N$ and $M$ be two $S$-sequences. The sequence $M$ is of type $N$ if

$$
N\left(s_{1}, \ldots, s_{n} ; s_{n+1}\right)=\varnothing \quad \Rightarrow \quad M\left(s_{1}, \ldots, s_{n} ; s_{n+1}\right)=\varnothing
$$


Proposition 1.9 Let $M$ be an $\{o ; c\}$-sequence of type Act. The following assertions are equivalent:

(i) $\quad M$ is an Act $_{>0}$-infinitesimal bimodule;

(ii) the families $M_{c}$ and $M_{o}$ are semicosimplicial spaces, and there exists a semicosimplicial map $h: M_{c} \rightarrow M_{o}$.

Moreover, (i) $\Rightarrow$ (ii) even if $M$ is not of type Act.

Proof Let $M$ be an $\mathcal{A} t_{>0}$-infinitesimal bimodule. For $n \in \mathbb{N}$, let $h: M_{c}^{n} \rightarrow M_{o}^{n}$ be defined by $h(x)=*_{2 ; o} \circ_{1} x$. The semicosimplicial structure is given as follows (see eg $[1 ; 15 ; 17])$ :

$$
\begin{aligned}
& d^{i}: M_{c}^{n} \rightarrow M_{c}^{n+1} ; x \mapsto \begin{cases}*_{2 ; c} \circ_{2} x & \text { if } i=0, \\
x \circ *_{2 ; c} & \text { if } i \in\{1, \ldots, n\}, \\
*_{2 ; c} \circ_{1} x & \text { if } i=n+1,\end{cases} \\
& d^{i}: M_{o}^{n} \rightarrow M_{o}^{n+1} ; \quad x \mapsto \begin{cases}*_{2 ; o} \circ_{2} x & \text { if } i=0, \\
x \circ *_{2 ; c} & \text { if } i \in\{1, \ldots, n\}, \\
x \circ{ }^{n+1} *_{2 ; o} & \text { if } i=n+1 .\end{cases}
\end{aligned}
$$

The reader can check that the relations (1) of Definition 1.4 and Definition 1.6 induce the semicosimplicial relations.

Conversely, if $h: M_{c} \rightarrow M_{o}$ is a semicosimplicial map, then let $M(n ; c)=M_{c}^{n}$, $M(n+1 ; o)=M_{o}^{n}$ and the empty set otherwise. The left and right infinitesimal module structures are defined by the above construction since $\mathcal{A c t}_{>0}$ is generated by $*_{2 ; c}$ and $*_{2 ; o}$ as a colored operad.

It is proved in [19] that the category of semicosimplicial spaces is equivalent to the category of As $>0$-infinitesimal bimodules. Consequently, the collection $M_{o}=\left\{M_{o}^{n}\right\}_{n \geq 0}$ is an infinitesimal bimodule over $\mathrm{As}_{>0}$. Since $\mathrm{As}_{>0}$ is generated by $*_{2}$ as an operad, the structure of $M_{o}$ is given by:

$$
\begin{cases}*_{2} \circ_{2} x=*_{2 ; o} \circ_{2} x, & \text { for } x \in M_{o}^{n}, \\ *_{2} \circ_{1} x=x \circ^{n+1} *_{2 ; o}, & \text { for } x \in M_{o}^{n}, \\ x \circ^{i} *_{2}=x \circ^{i} *_{2 ; c}, & \text { for } x \in M_{o}^{n} \text { and } i \in\{1, \ldots, n\} .\end{cases}
$$

\section{C Bimodules over a colored operad}

Definition 1.10 Let $O$ be an $S$-operad. An $S$-sequence $M$ is an $O$-bimodule if it 
is endowed with operations

$$
\begin{aligned}
\gamma_{l}: O\left(s_{1}, \ldots, s_{n} ; s_{n+1}\right) \times M\left(s_{1}^{1}, \ldots, s_{p_{1}}^{1} ; s_{1}\right) \times \cdots \times M\left(s_{1}^{n}, \ldots, s_{p_{n}}^{n} ; s_{n}\right) & \\
\circ^{i}: M\left(s_{1}, \ldots, s_{n} ; s_{n+1}\right) \times & \rightarrow M\left(s_{1}^{1}, \ldots, s_{p_{n}}^{n} ; s_{n+1}^{\prime}, \ldots, s_{m}^{\prime} ; s_{i}\right), \\
& \rightarrow M\left(s_{1}, \ldots, s_{i-1}, s_{1}^{\prime}, \ldots, s_{m}^{\prime}, s_{i+1}, \ldots, s_{n} ; s_{n+1}\right),
\end{aligned}
$$

for $1 \leq i \leq n$, satisfying associativity and unit axioms [1]. A map between $O$-bimodules is an $S$-sequence map that preserves the bimodule structure. Let $\operatorname{Bimod}_{O}$ be the category of $O$-bimodules. We denote by $x\left(y_{1}, \ldots, y_{n}\right)$ the operation $\gamma_{l}\left(x, y_{1}, \ldots, y_{n}\right)$ with $x \in O$ and $y_{i} \in M$.

Example 1.11 If $\eta: O_{1} \rightarrow O_{2}$ is any $S$-operad map, then $O_{2}$ is endowed with the following $O_{1}$-bimodule structure:

$$
\gamma_{l}: O_{1} \times O_{2} \times \cdots \times O_{2} \stackrel{\eta \times \text { id } \times \cdots \times \text { id }}{\longrightarrow} O_{2} \times \cdots \times O_{2} \rightarrow O_{2}
$$

and

$$
\circ^{i}: O_{2} \times O_{1} \stackrel{\mathrm{id} \times \eta}{\longrightarrow} O_{2} \times O_{2} \rightarrow O_{2} .
$$

Consequently, if $A$ is an $O$-algebra, then $\operatorname{End}_{A}$ is an $O$-bimodule.

A priori there is no relation between an $O$-bimodule structure and an $O$-infinitesimal bimodule structure because the left operations differ. However, if $\eta: O \rightarrow M$ is a morphism of $O$-bimodules, then $M$ is an $O$-infinitesimal bimodule, and the left infinitesimal bimodule structure is given by

$$
\begin{aligned}
\circ_{i}: O\left(s_{1}, \ldots, s_{n} ; s_{n+1}\right) \times M\left(s_{1}^{\prime}\right. & \left., \ldots, s_{m}^{\prime} ; s_{i}\right) \\
& \rightarrow M\left(s_{1}, \ldots, s_{i-1}, s_{1}^{\prime}, \ldots, s_{m}^{\prime}, s_{i+1}, \ldots, s_{n} ; s_{n+1}\right), \\
(o ; m) & \mapsto o\left(\eta\left(*_{s_{1}}\right), \ldots, \eta\left(*_{s_{i-1}}\right), m, \eta\left(*_{s_{i+1}}\right), \ldots, \eta\left(*_{s_{n}}\right)\right),
\end{aligned}
$$

where $*_{s}$ is the distinguished element in $O(s ; s)$.

In [15], McClure and Smith define a monoidal structure on the category of semicosimplicial spaces in order to recognize loop spaces. More precisely, they prove that the group completion of the semitotalization of a monoid in this category has the homotopy type of a loop space. We recall this construction since we need it to describe $\mathcal{A} c t_{>0}$-bimodules under $\mathcal{A} c t$.

Proposition 1.12 [15, Proposition 2.2] Let $X^{\bullet}$ and $Y^{\bullet}$ be two semicosimplicial spaces, and let $X \otimes Y$ be the semicosimplicial space whose $m^{\text {th }}$ space is given by

$$
\left(\coprod_{p+q=m} X^{p} \times Y^{q}\right) / \sim,
$$


where $\sim$ is the equivalence relation generated by $\left(x, d^{0} y\right) \sim\left(d^{|x|+1} x, y\right)$. The semicosimplicial structure is the following:

$$
d^{i}(x, y)= \begin{cases}\left(d^{i} x, y\right) & \text { if } 0 \leq i \leq|x| \\ \left(x, d^{i-|x|} y\right) & \text { if }|x|<i \leq|x|+|y|+1 .\end{cases}
$$

The category of semicosimplicial spaces equipped with $\otimes$ is a monoidal category de-

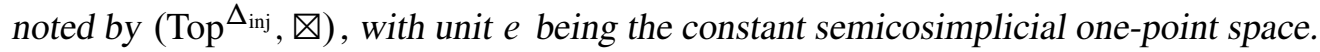

Proposition 1.13 Let $M$ be an $\{o ; c\}$-sequence of type $\mathcal{A} c t$. The following assertions are equivalent:

(i) $\quad M$ is an $\mathcal{A c t}_{>0}$-bimodule under $\mathcal{A}$ ct;

(ii) in (Top $\left.{ }^{\Delta_{\text {inj }}}, \nabla\right)$, the family $M_{c}$ is a monoid with unit, the family $M_{o}$ is an $M_{c}-$ left module and there exists a morphism of $M_{c}$-left modules $h: M_{c} \rightarrow M_{o}$.

Moreover (i) $\Rightarrow$ (ii) even if $M$ is not of type $\mathcal{A c t}$.

Proof Let $M$ be an $\mathcal{A} c t_{>0}$-bimodule equipped with an $\mathcal{A c t}_{>0}$-bimodule map $\eta: \mathcal{A c t} \rightarrow M$. Let $M_{c}^{n}=M(n ; c)$ and $M_{o}^{n}=M(n+1 ; o)$ for $n \in \mathbb{N}$. The bimodule structure induces, for $i \in\{1, \ldots, n\}$, the cofaces

$$
\begin{array}{lll}
d^{i}: M_{c}^{n} \rightarrow M_{c}^{n+1}, & x \mapsto x \circ^{i} *_{2 ; c}, \\
d^{i}: M_{o}^{n} \rightarrow M_{o}^{n+1}, & x \mapsto x \circ^{i} *_{2 ; c}, \\
d^{n+1}: M_{o}^{n} \rightarrow M_{o}^{n+1}, & x \mapsto x \circ^{n+1} *_{2 ; o},
\end{array}
$$

satisfying the semicosimplicial relations and the following two operations:

$$
\begin{array}{ll}
M_{c}^{j} \times M_{c}^{l} \rightarrow M_{c}^{j+l}, & (x ; y) \mapsto *_{2 ; c}(x ; y), \\
M_{c}^{j} \times M_{o}^{l} \rightarrow M_{o}^{j+l}, & (x ; y) \mapsto *_{2 ; o}(x ; y) .
\end{array}
$$

The map $\eta: \mathcal{A} c t \rightarrow M$ gives us the missing cofaces

$$
\begin{array}{lll}
d^{0}: M_{c}^{n} \rightarrow M_{c}^{n+1}, & x \mapsto *_{2 ; c}\left(\eta\left(*_{1 ; c}\right), x\right), \\
d^{n+1}: M_{c}^{n} \rightarrow M_{c}^{n+1}, & x \mapsto *_{2 ; c}\left(x, \eta\left(*_{1 ; c}\right)\right), \\
d^{0}: M_{o}^{n} \rightarrow M_{o}^{n+1}, & x \mapsto *_{2 ; o}\left(\eta\left(*_{1 ; c}\right), x\right),
\end{array}
$$

inducing a semicosimplicial structure on $M_{c}$ and $M_{o}$ such that the two operations defined in (3) make $M_{c}$ into a monoid with unit and $M_{o}$ into an $M_{c}$-left module. The map

$$
h: M_{c}^{n} \rightarrow M_{o}^{n}, \quad x \mapsto *_{2 ; o}\left(x, \eta\left(*_{1 ; o}\right)\right)
$$

is a left $M_{c}$-module map. 
Conversely, let $\left(M_{c}, M_{o}, h\right)$ be a triple satisfying the conditions of the proposition. By the same argument as in Proposition 1.9, the constructions (3) and (4) define an $\mathcal{A} c t_{>0}$-bimodule structure on $M$. In particular, if $M_{c}$ and $M_{o}$ coincide with the unit $e$, then the corresponding $\mathcal{A} c t_{>0}$-bimodule is $\mathcal{A} c t$. There exists a map $\eta_{c}$ from the unit to $M_{c}$, for $M_{c}$ is a monoid with unit. Let $\eta_{o}$ be the map from the unit to $M_{o}$ given by $\eta_{o}=h \circ \eta_{c}$. The map $\eta: \mathcal{A} c t \rightarrow M$ so obtained is an $\mathcal{A} c t_{>0}$-bimodule map.

Example 1.14 This proposition implies that the category whose objects are monoids in $\left(\mathrm{Top}^{\Delta_{\text {inj }}}, \nabla\right)$ is equivalent to the category of $\mathrm{As}_{>0}$-bimodules under As considered by Turchin. Furthermore, if we substitute $\mathcal{A c t}_{>0}$-bimodule by $\mathcal{A} c t$-bimodule and semicosimplicial space by cosimplicial space, Proposition 1.13 is still true. From now on, $(X ; *)$ is a pointed topological space, and $A$ is a subspace of $X$ containing the basepoint $*$. Let $\Omega X^{\bullet}$ and $\Omega(X ; A)^{\bullet}$ be the two cosimplicial spaces defined respectively by

$$
\Omega X^{n}:=X^{\times n} \quad \text { and } \quad \Omega(X ; A)^{n}:=X^{\times n} \times A \text { for } n \in \mathbb{N},
$$

and

$$
d^{i}: \Omega X^{n} \rightarrow \Omega X^{n+1}, \quad\left(x_{1}, \ldots, x_{n}\right) \mapsto \begin{cases}\left(*, x_{1}, \ldots, x_{n}\right) & \text { if } i=0, \\ \left(x_{1}, \ldots, x_{i}, x_{i}, \ldots, x_{n}\right) & \text { if } i \in\{1, \ldots, n\}, \\ \left(x_{1}, \ldots, x_{n}, *\right) & \text { if } i=n+1,\end{cases}
$$

$d^{i}: \Omega(X ; A)^{n} \rightarrow \Omega(X ; A)^{n+1}$,

$$
\left(x_{1}, \ldots, x_{n}, a\right) \mapsto \begin{cases}\left(*, x_{1}, \ldots, x_{n}, a\right) & \text { if } i=0, \\ \left(x_{1}, \ldots, x_{i}, x_{i}, \ldots, x_{n}, a\right) & \text { if } i \in\{1, \ldots, n\}, \\ \left(x_{1}, \ldots, x_{n}, a, a\right) & \text { if } i=n+1 .\end{cases}
$$

The codegeneracies consist in forgetting a point, and the concatenation makes $\Omega X^{\bullet}$ into a monoid with unit in (Top $\left.{ }^{\Delta_{\text {inj }}}, \nabla\right)$, and $\Omega(X ; A)^{\bullet}$ into a left $\Omega X^{\bullet}$-module. The left $\Omega X^{\bullet}$-module map is defined by

$$
h: \Omega X^{n} \rightarrow \Omega(X ; A)^{n}, \quad\left(x_{1}, \ldots, x_{n}\right) \mapsto\left(x_{1}, \ldots, x_{n}, *\right) .
$$

Proposition 1.13 states that these data are equivalent to an $\mathcal{A c t}$-bimodule map. So the pair $\left(\Omega X^{\bullet} ; \Omega(X ; A)^{\bullet}\right)$ is the prototypical example of an $\mathcal{A} t$-bimodule. The evaluation maps,

$$
\begin{array}{lll}
\Omega X \rightarrow \operatorname{Tot}\left(\Omega X^{\bullet}\right), & f \mapsto\left\{f_{n}:\left(t_{1} ; \cdots ; t_{n}\right) \mapsto\left(f\left(t_{1}\right), \ldots, f\left(t_{n}\right)\right)\right\}_{n}, \\
\Omega(X ; A) \rightarrow \operatorname{Tot}\left(\Omega(X ; A)^{\bullet}\right), & f \mapsto\left\{f_{n}:\left(t_{1} ; \cdots ; t_{n}\right) \mapsto\left(f\left(t_{1}\right), \ldots, f\left(t_{n}\right), f(1)\right)\right\}_{n},
\end{array}
$$

induce homeomorphisms, due to the codegeneracies. Here $\Omega X$ and $\Omega(X ; A)$ are the space of loops based on $*$ and the space of paths with endpoint in the subspace 
$A$, respectively, and $\left(t_{1} ; \cdots ; t_{n}\right)$ is a nondecreasing sequence; that is, $t_{i} \leq t_{i+1}$ for $1 \leq i<n$. As seen in the introduction, these two spaces are models for the homotopy fiber $* \rightarrow X$ and $A \rightarrow X$ over the basepoint $*$, respectively.

It provides an example of an $\mathcal{A} c t$-bimodule map $\eta: \mathcal{A} t \rightarrow M$ such that the totalization of $M_{c}$ (resp. $M_{o}$ ) can be described as a loop space $\Omega X$ (resp. a relative loop space $\Omega(X ; A))$ with explicit topological spaces $X$ and $A$. We will prove that we can generalize this result for any $\mathcal{A} c t_{>0}$-bimodule map $\eta$ : $\mathcal{A c t} \rightarrow M$ using the semitotalization. Let us notice that, in this particular case, the sequence of cosimplicial spaces $\Omega A^{\bullet} \rightarrow \Omega X^{\bullet} \rightarrow \Omega(X ; A)^{\bullet} \rightarrow A \rightarrow X$ gives rise, under totalization, to the dual of the Barratt-Puppe sequence for the map $A \rightarrow X$.

\section{The free (infinitesimal) bimodule generated by an $S$-sequence}

In what follows, $S$ is a set, $O$ is an $S$-operad and $M$ is an $S$-sequence. In order to prove that $\operatorname{sTot}\left(M_{o}\right)$ has the homotopy type of a relative loop space and to explicitly identify this space, we have to introduce a model category structure on the categories $\operatorname{Ibimod}_{O}$ and $\operatorname{Bimod}_{O}$. The easiest way is to use a transfer theorem (see eg Theorem 3.4), which needs a left adjoint to the forgetful functor from the category of (infinitesimal) bimodules over $O$ to $\operatorname{Coll}(S)$. In both cases, the first step consists in introducing the category of trees, which encodes the (infinitesimal) bimodule structure. Then we label the vertices by points in $M$ or $O$. Similar constructions have been considered in [5] and, more recently, [20].

By a tree we mean a planar rooted tree with an orientation towards the root, and let $t$ be such a tree.

- The set of its vertices is denoted by $V(t)$ and the set of its edges by $E(t)$.

- For a vertex $v$, the ordered set of its input edges is denoted by $\operatorname{in}(v)$ and its cardinality by $|v|$ such that in $(v)=\left\{e_{1}(v), \ldots, e_{|v|}(v)\right\}$. The output edge of $v$ is denoted by $e_{0}(v)$, and a vertex without input edge is called univalent.

- The edges connecting two vertices are called inner edges, and the set of inner edges is denoted by $E^{\text {int }}(t)$.

- An element $e \in E^{\mathrm{int}}(t)$ is determined by a source vertex $s(e)$ and a target vertex $t(e)$ induced by the orientation of the tree.

- An edge with no source is called a leaf, and the ordered set of leaves is denoted by $\left\{l_{1}, \ldots, l_{n}\right\}$. 
- The edge with no target is called the trunk, denoted by $e_{0}$, and its source, the root, is denoted by $r$.

- Each leaf is connected to the trunk by a unique path composed of edges.

- An $S$-tree is a pair $(t, f)$ where $t$ is a planar tree and $f: E(t) \rightarrow S$ is called an $S$-labeling of $t$.

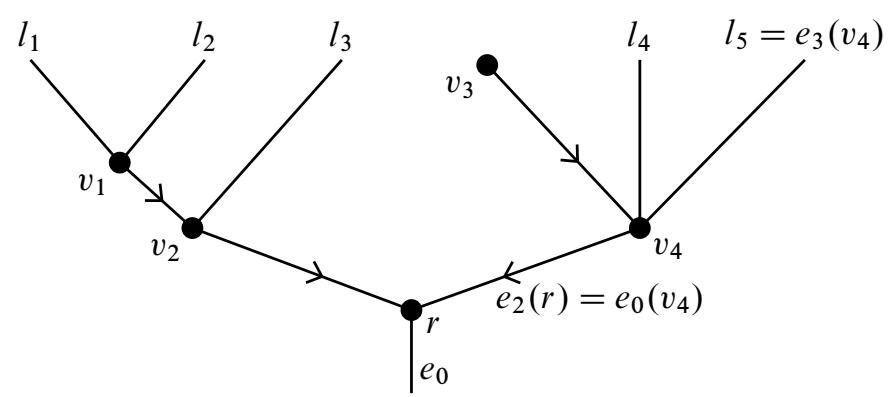

Figure 1: A planar tree: $r$ is the root, $e_{0}$ is the trunk, $l_{1}$ is a leaf.

\section{A The free infinitesimal bimodule}

Definition 2.1 The trees encoding the infinitesimal bimodule structure are constructed as follows.

- The join $j\left(v_{1} ; v_{2}\right)$ of two vertices $v_{1}$ and $v_{2}$ is the first common vertex shared by the two paths joining $v_{1}$ and $v_{2}$ to the root. If $j\left(v_{1} ; v_{2}\right)=r$, then $v_{1}$ and $v_{2}$ are said to be connected to the root, and if $j\left(v_{1} ; v_{2}\right) \in\left\{v_{1} ; v_{2}\right\}$, then they are said to be connected. In Figure 1, the vertices $v_{1}$ and $v_{2}$ are connected whereas the vertices $v_{1}$ and $v_{3}$ are connected to the root.

- Let $d: V(T) \times V(T) \rightarrow \mathbb{N}$ be the distance defined as follows. The integer $d\left(v_{1} ; v_{2}\right)$ is the number of edges connecting $v_{1}$ to $v_{2}$ if they are connected, otherwise $d\left(v_{1} ; v_{2}\right)=d\left(v_{1} ; v_{3}\right)+d\left(v_{3} ; v_{2}\right)$ with $v_{3}=j\left(v_{1} ; v_{2}\right)$. In Figure 1 , $d\left(v_{1} ; r\right)=2, d\left(v_{3} ; v_{4}\right)=1$ and $d\left(v_{1} ; v_{3}\right)=4$.

- A pearl tree (or ptree) is a pair $(t, p)$ where $t$ is a planar tree and $p \in V(t)$ is called the pearl, satisfying the property: if $v \in V(t) \backslash\{p\}$, then $d(v ; p)=1$. An $S$-ptree is a pearl tree $t$ together with an $S$-labeling of $t$; see Figure 2. 


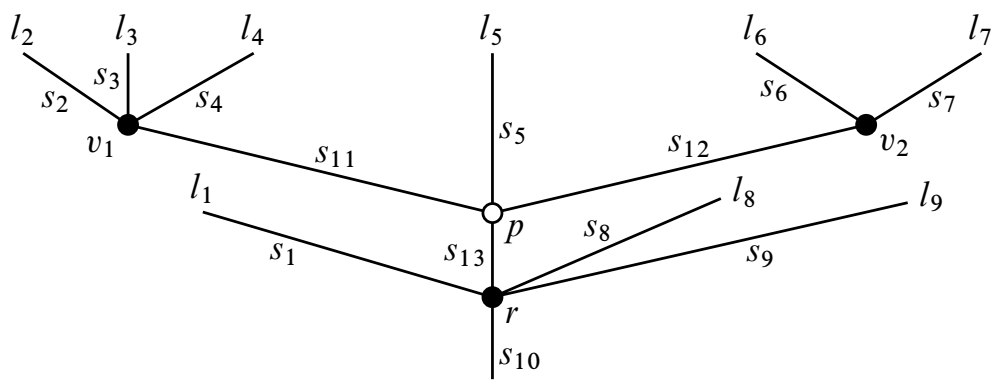

Figure 2: An $S$-ptree.

Construction 2.2 The $S$-sequence $\operatorname{Ib}_{O}(M)$ is defined as follows:

$\mathrm{Ib}_{O}(M)\left(s_{1}, \ldots, s_{n} ; s_{n+1}\right)$

$$
\begin{aligned}
= & \coprod_{\substack{(t, f, p) \in S \text {-ptree } \\
f\left(l_{i}\right)=s_{i}, f\left(e_{0}\right)=s_{n+1}}}\left[M\left(f\left(e_{1}(p)\right), \ldots, f\left(e_{|p|}(p)\right) ; f\left(e_{0}(p)\right)\right)\right. \\
& \left.\times \prod_{v \in V(t) \backslash\{p\}} O\left(f\left(e_{1}(v)\right), \ldots, f\left(e_{|v|}(v)\right) ; f\left(e_{0}(v)\right)\right)\right] / \sim
\end{aligned}
$$

Here, $\sim$ is the equivalence relation generated by

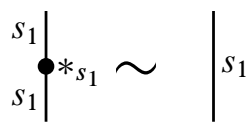

Let $x$ be a point in the space $\operatorname{Ib}_{O}(M)\left(s_{1}, \ldots, s_{n} ; s_{n+1}\right)$ indexed by an $S$-ptree $(t, f, p)$, and let $y \in O\left(s_{1}^{\prime}, \ldots, s_{m}^{\prime} ; s_{i}\right)$. The right infinitesimal module structure consists of grafting the $m$-corolla indexed by $y$ to the $i^{\text {th }}$ input of $t$, and contracting the inner edge so obtained if its target does not coincide with the pearl, by using the operadic structure of $O$, as in Figure 3.
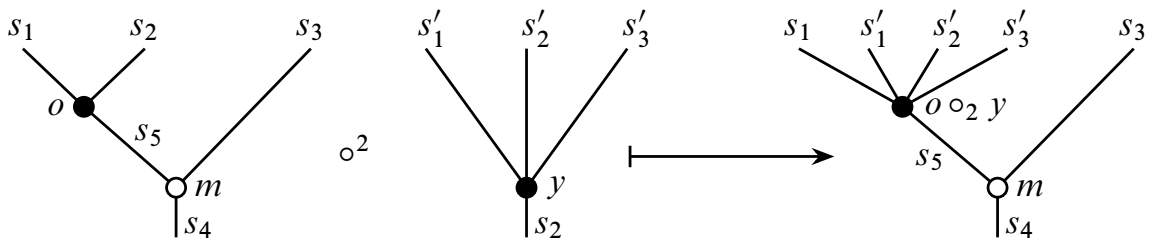

Figure 3: The right infinitesimal module structure.

Similarly, let $x$ be a point in the space $\operatorname{Ib}_{O}(M)\left(s_{1}^{\prime}, \ldots, s_{m}^{\prime} ; s_{i}\right)$ indexed by an $S-$ ptree $(t, f, p)$ and let $y \in O\left(s_{1}, \ldots, s_{n} ; s_{n+1}\right)$. The left infinitesimal module structure consists in grafting the tree $t$ to the $i^{\text {th }}$ input of the $n$-corolla indexed by $y$, and 
contracting the inner edge so obtained if its source does not coincide with the pearl, by using the operadic structure of $O$. These maps pass to the quotient and are continuous. There exists a map from the $S$-sequence $M$ to $\operatorname{Ib}_{O}(M)$ that maps a point $m \in$ $M\left(s_{1}, \ldots, s_{n} ; s_{n+1}\right)$ to the pearl $n$-corolla whose leaves are labeled by $s_{1}, \ldots, s_{n}$ and the trunk by $s_{n+1}$; the pearl is indexed by $m$.

We denote by $(t, f, p, g)$ a point in $\operatorname{Ib}_{O}(M)$ indexed by $(t, f, p)$ and labeled by $g: V(t) \rightarrow O \sqcup M$.

Proposition 2.3 The functor $\mathrm{Ib}_{O}$ is the left adjoint to the forgetful functor:

$$
\operatorname{Ib}_{O}(-): \operatorname{Coll}(S) \leftrightarrows \operatorname{Ibimod}_{O}: U
$$

Proof Given an $O$-infinitesimal bimodule $N$ and a map of $S$-sequences $h: M \rightarrow N$, we prove that there exists a unique map $\tilde{h}: \mathrm{Ib}_{O}(M) \rightarrow N$ of $O$-infinitesimal bimodules such that the following diagram commutes:

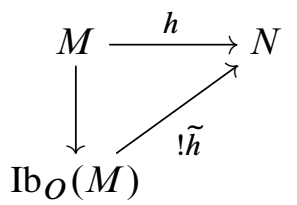

Let $(t, f, p, g)$ be a point in $\operatorname{Ib}_{O}(M)$. The map $\tilde{h}$ is defined by induction on $|V(t)|$ as follows. If $|V(t)|=1$, then the pearl $p$ is the only vertex and $t$ is a corolla. In this case we define $\tilde{h}((t, f, p, g))=h(g(p))$. Hence the commutativity of the previous diagram is guaranteed.

If $t$ has two vertices, then there exists a unique edge $e$ connecting the pearl $p$ to the other vertex $v$. There are two cases to consider:

- if $s(e)=p$ and $e$ is the $i^{\text {th }}$ input of $v$, then let $\tilde{h}((t, f, p, g))=g(v) \circ_{i} h(g(p))$;

- if $t(e)=p$ and $e$ is the $i^{\text {th }}$ input of $p$, then let $\tilde{h}((t, f, p, g))=h(g(p)) \circ^{i} g(v)$.

Assume $\tilde{h}$ has been defined for $|V(t)|=n \geq 2$. Let $(t, f, p, g) \in \operatorname{Ib}_{O}(M)$ such that $t$ has $n+1$ vertices. There exists an inner edge $e$ connecting the pearl $p$ to another vertex $v$ such that $t(e)=p$. Let $\left(t^{\prime}, f^{\prime}, p, g^{\prime}\right)$ be the tree obtained by cutting off the corolla corresponding to the vertex $v$ ( $t^{\prime}$ has only $n$ vertices ). We define

$$
\widetilde{h}((t, f, p, g))=\tilde{h}\left(\left(t^{\prime}, f^{\prime}, p, g^{\prime}\right)\right) \circ^{i} g(v) .
$$

Due to the associativity axioms of the infinitesimal bimodule structure of $N, \tilde{h}$ does not depend on the choice of $v$, and $\tilde{h}$ is an infinitesimal bimodule map. The uniqueness follows from the construction. 


\section{B The free bimodule}

Definition 2.4 A tree with section (or stree) is a pair $\left(t, V^{p}(t)\right)$ where $t$ is a planar tree and $V^{p}(t)$ is a subset of $V(t)$, called the set of pearls, such that each path connecting a leaf or an univalent vertex to the trunk passes by a unique pearl, and

$$
j(v ; p) \in\{v ; p\} \quad \Rightarrow \quad d(v ; p)=1
$$

for all $v \in V(t) \backslash V^{p}(t)$ and all $p \in V^{p}(t)$. An $S$-tree with section (or $S$-tree) is given by a triple $\left(t, V^{p}(t), f\right)$ such that $(t, f)$ is an $S$-tree and $\left(t, V^{p}(t)\right)$ is a tree with section.

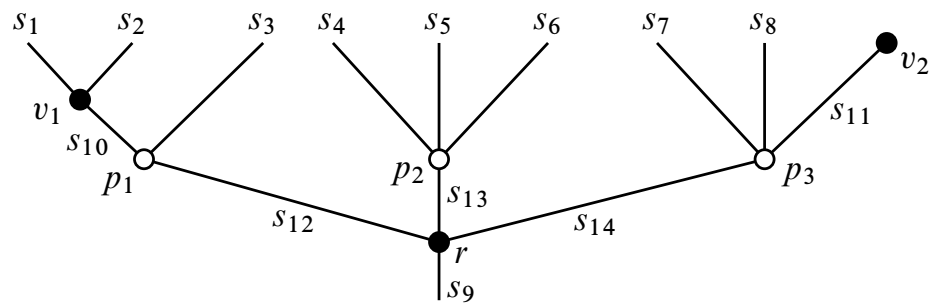

Figure 4: A tree with section.

Construction 2.5 The $S$-sequence $B_{O}(M)$ is defined as follows:

$$
\begin{aligned}
B_{O}(M)\left(s_{1}, \ldots, s_{n} ; s_{n+1}\right) & \\
= & \coprod_{\substack{\left(t, f, V^{p}(t)\right) \in S \text {-stree } \\
f\left(l_{i}\right)=s_{i}, f\left(e_{0}\right)=s_{n+1}}}\left[\prod_{v \in V^{p}(t)} M\left(f\left(e_{1}(v)\right), \ldots, f\left(e_{|v|}(v)\right) ; f\left(e_{0}(v)\right)\right)\right. \\
& \left.\times \prod_{v \in V(t) \backslash V^{p}(t)} O\left(f\left(e_{1}(v)\right), \ldots, f\left(e_{|v|}(v)\right) ; f\left(e_{0}(v)\right)\right)\right] / \sim
\end{aligned}
$$

with $\sim$ the equivalence relation generated by

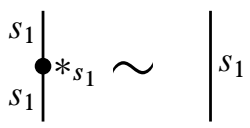

Let $x \in B_{O}(M)\left(s_{1}, \ldots, s_{n} ; s_{n+1}\right)$ be indexed by a tree with section $\left(t, f, V^{p}(t)\right)$, and let $y \in O\left(s_{1}, \ldots, s_{n} ; s_{i}\right)$. The right module structure consists of grafting the $m$-corolla indexed by $y$ to the $i^{\text {th }}$ input of $t$, and contracting the inner edge so obtained if its target does not coincide with a pearl, by using the operadic structure of $O$.

Let $y$ be a point in $O\left(s_{1}, \ldots, s_{n} ; s_{n+1}\right)$ and let $x_{i} \in B_{O}(M)\left(s_{1}^{i}, \ldots, s_{n_{i}}^{i} ; s_{i}\right)$ be indexed by $\left(t_{i}, f_{i}, V_{i}^{p}(t)\right)$ for $1 \leq i \leq n$. The left module structure consists of grafting each 
tree $t_{i}$ to the $i^{\text {th }}$ input of the $n$-corolla indexed by $y$, and contracting the inner edges whose source is not a pearl by using the operadic structure of $O$, as in Figure 5.

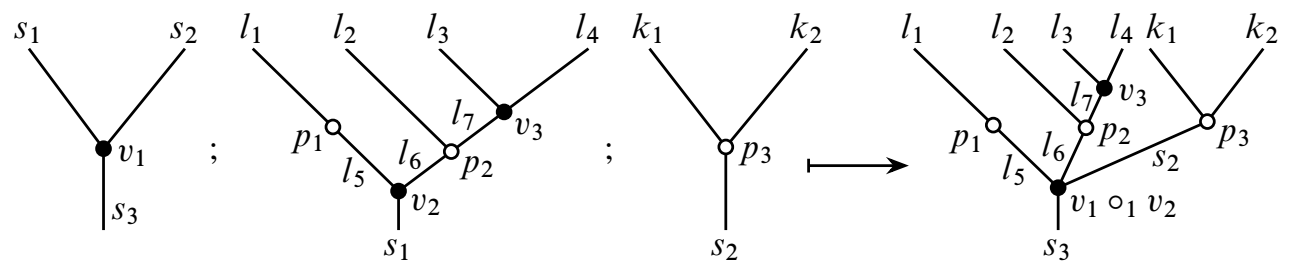

Figure 5: The left module structure.

These maps pass to the quotient and are continuous. Furthermore, there exists a map from the $S$-sequence $M$ to $B_{O}(M)$ which maps a point $m \in M\left(s_{1}, \ldots, s_{n} ; s_{n+1}\right)$ to the pearl $n$-corolla whose leaves are labeled by $s_{1}, \ldots, s_{n}$ and the trunk by $s_{n+1}$; the pearl is indexed by $m$. We denote by $\left(t, f, V^{p}(t), g\right)$ a point in $B_{O}(M)$ indexed by $\left(t, f, V^{p}(t)\right)$ and labeled by $g: V(t) \rightarrow O \sqcup M$.

Proposition 2.6 The functor $B_{O}$ is the left adjoint to the forgetful functor:

$$
B_{O}(-): \operatorname{Coll}(S) \leftrightarrows \operatorname{Bimod}_{O}: U
$$

Proof Given an $O$-bimodule $N$ and $h: M \rightarrow N$ a map of $S$-sequences, we prove that there exists a unique map $\tilde{h}: B_{O}(M) \rightarrow N$ of $O$-bimodules such that the following diagram commutes:

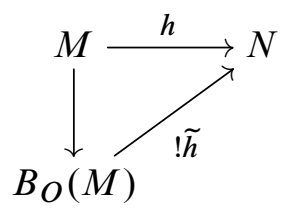

Let $\left(t, V^{p}(t), f, g\right)$ be a point in $B_{O}(M)$ and let $\mathrm{nb}(t)$ be the cardinality of the set $V(t) \backslash V^{p}(t)$. The map $\tilde{h}$ is defined by induction on $\operatorname{nb}(t)$. If $\operatorname{nb}(t)=0$, then the pearl $p$ is the only vertex, and $t$ is a corolla. In this case, $\tilde{h}\left(\left(t, V^{p}(t), f, g\right)\right)=h(g(p))$. If $\mathrm{nb}(t)=1$, we denote by $v$ the unique element of $V(t) \backslash V^{p}(t)$. There are two cases to consider.

- If $v$ is the source of an edge $e$ that is connected to a pearl $p$, and $e$ is the $i^{\text {th }}$ input of $p$, then

$$
\tilde{h}\left(\left(t, V^{p}(t), f, g\right)\right)=h(g(p)) \circ^{i} g(v) .
$$

- If $v$ coincides with the root, then all the pearls are connected to $v$. Let $p_{1}, \ldots, p_{k}$ be the set of ordered pearls. We define $\tilde{h}$ by

$$
\tilde{h}\left(\left(t, V^{p}(t), f, g\right)\right)=g(v)\left(h\left(g\left(p_{1}\right)\right), \ldots, h\left(g\left(p_{k}\right)\right)\right) .
$$


Assume $\tilde{h}$ has been defined for $\operatorname{nb}(t)=n \geq 1$. Let $\left(t, V^{p}(t), f, g\right) \in B_{O}(M)$ such that $\mathrm{nb}(t)=n+1$. There exists an inner edge $e$ whose target is a pearl $p_{i}$. Let $v=s(e)$ and let $\left(t^{\prime}, V^{p}(t), f^{\prime}, g^{\prime}\right)$ be the tree obtained from $\left(t, V^{p}(t), f, g\right)$ by cutting off the corolla corresponding to the vertex $v$. Consequently, $\operatorname{nb}\left(t^{\prime}\right)=n$ and $\widetilde{h}$ can be defined by induction as

$$
\tilde{h}\left(\left(t, V^{p}(t), f, g\right)\right)=\tilde{h}\left(\left(t^{\prime}, V^{p}(t), f^{\prime}, g^{\prime}\right)\right) \circ^{i} g(v) .
$$

Due to the associativity axioms of the bimodule structure of $N, \tilde{h}$ does not depend on the choice of $v$, and $\tilde{h}$ is a map of $O$-bimodules. The uniqueness follows from the construction.

\section{Cofibrant replacement of the operad of monoid actions in the category of (infinitesimal) bimodules over $\mathcal{A c t}_{>0}$}

\section{A Model category structure on $\operatorname{Bimod}_{O}$ and Ibimod $o$}

In this section we define a model category structure on $\operatorname{Bimod} O$ and $\operatorname{Ibimod} O$ by using the previous adjunctions. The references used for model categories are $[8 ; 12 ; 14]$. These structures have been considered by many authors in the context of operads (symmetric or nonsymmetric), algebras over an operad, or left-right modules over operads, most of them in the uncolored case; see eg Fresse [9], Berger and Moerdijk [2] and Harper [10]. In order to be precise, we prefer to give the details of the model category structure in our context, and we make use of this section to state lemmas that will be useful for the sequel.

Theorem 3.1 [14, Theorem 2.4.24] The category Top is equipped with the following model category structure.

Weak equivalences are the continuous maps $f: X \rightarrow Y$ such that $f_{0}^{*}: \pi_{0}(X) \rightarrow$ $\pi_{0}(Y)$ is a bijection and $f_{n}^{*}: \pi_{n}(X ; x) \rightarrow \pi_{n}(Y ; f(x))$ is an isomorphism, for all $x \in X$ and for all $n>0$.

Serre fibrations are the continuous maps $f: X \rightarrow Y$ having the homotopy lifting property, ie for every $C W$-complex $A$, a lift exists in every commutative diagram of the following form:

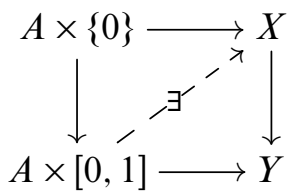


Cofibrations are the continuous maps having the left lifting property with respect to the acyclic Serre fibrations.

Moreover, this model category is cofibrantly generated. The cofibrations are generated by the inclusions $\partial \Delta^{n} \rightarrow \Delta^{n}$ for $n>0$, whereas the acyclic cofibrations are generated by the inclusions of the horns $\Lambda_{k}^{n} \rightarrow \Delta^{n}$ for $n>0$ and $n \geq k \geq 0$. We call this model category the Serre model category.

Corollary 3.2 The category Coll $(S)$ inherits a cofibrantly generated model category structure from the Serre model category in which a map is a cofibration, a fibration or a weak equivalence if each of its components is.

Lemma 3.3 [12] Let $A \hookrightarrow B$ be a cofibration in the Serre model category. For every space $Y$, the induced map $\operatorname{Top}(B ; Y) \rightarrow \operatorname{Top}(A ; Y)$ is a fibration.

Theorem 3.4 [2, Section 2.5] Let $\mathcal{C}_{1}$ be a cofibrantly generated model category and let $I$ (resp. $J$ ) be the set of generating cofibrations (resp. acyclic cofibrations). Let $L: \mathcal{C}_{1} \leftrightarrows \mathcal{C}_{2}: R$ be a pair of adjoint functors. Assume that $\mathcal{C}_{2}$ has small colimits and small limits. Define a map $f$ in $\mathcal{C}_{2}$ to be a weak equivalence (resp. a fibration) if $R(f)$ is a weak equivalence (resp. fibration). If we have that

(i) the functor $R$ preserves filtered colimits,

(ii) $\mathcal{C}_{2}$ has a functorial fibrant replacement, and

(iii) for each fibrant object $X \in \mathcal{C}_{2}$ we have a functorial path object $\operatorname{Path}(X)$ with $X \stackrel{\simeq}{\rightarrow} \operatorname{Path}(X) \rightarrow X \times X$ (a weak equivalence followed by a fibration) a factorization of the diagonal map,

then $\mathcal{C}_{2}$ is equipped with a cofibrantly generated model category $(L I, L J)$ with $L I=$ $\{L(u) \mid u \in I\}$ and $L J=\{L(v) \mid v \in J\}$. Furthermore, $(L, R)$ is a Quillen pair.

Application 3.5 For the adjunction $\operatorname{Ib}_{O}(-): \operatorname{Coll}(S) \rightleftarrows \operatorname{Ibimod}_{O}: U$, the identity induces a functorial fibrant replacement since all the objects of $\operatorname{Coll}(S)$ are fibrants. From an $O$-infinitesimal bimodule $M$, a functorial path object $\operatorname{Path}(M)$ is given by the following $S$-sequence:

$$
\operatorname{Path}(M)\left(s_{1}, \ldots, s_{n} ; s_{n+1}\right)=\operatorname{Top}\left([0,1] ; M\left(s_{1}, \ldots, s_{n} ; s_{n+1}\right)\right) .
$$

The $O$-infinitesimal bimodule structure and the functoriality of Path(-) are induced by that of $M$. The factorization of the diagonal map is given pointwise:

$$
M \stackrel{f_{1}}{\longrightarrow} \operatorname{Path}(M) \stackrel{f_{2}}{\longrightarrow} M \times M .
$$


The map $f_{1}$ maps a point $m \in M\left(s_{1}, \ldots, s_{n} ; s_{n+1}\right)$ to the constant path in $m$. Due to the homotopy between a path $h$ and the constant path in $h(0)$, the map $f_{1}$ is a weak equivalence. The map $f_{2}$ maps a point $h \in \operatorname{Path}(M)\left(s_{1}, \ldots, s_{n} ; s_{n+1}\right)$ to the pair $(h(0) ; h(1)) \in(M \times M)\left(s_{1}, \ldots, s_{n} ; s_{n+1}\right)$. This map is a fibration since $\operatorname{Path}(M)\left(s_{1}, \ldots, s_{n} ; s_{n+1}\right)$ is a path object in the Serre model category.

Similarly, the adjunction $B_{O}(-)$ : $\operatorname{Coll}(S) \leftrightarrows \operatorname{Bimod}_{O}: U$ induces a cofibrantly generated model category on $\operatorname{Bimod} O$.

Definition 3.6 The $O$-infinitesimal bimodule $M$ is obtained from the $O$-infinitesimal bimodule $N$ by attaching cells if $M$ is obtained as a pushout diagram of the form

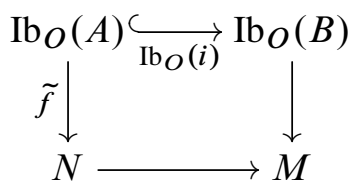

with $i$ a cofibration in $\operatorname{Coll}(S), f: A \rightarrow N$ an $S$-sequence map called the attaching map and $\tilde{f}$ the $O$-infinitesimal bimodule map induced by $f$; see Proposition 2.3.

Similarly, an $O$-bimodule $M$ is obtained from an $O$-bimodule $N$ by attaching cells if $M$ is obtained as a pushout diagram of the form

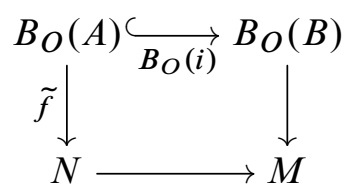

with $i$ a cofibration in $\operatorname{Coll}(S), f: A \rightarrow N$ an $S$-sequence map called the attaching map and $\tilde{f}$ the $O$-bimodule map induced by $f$; see Proposition 2.6. In both cases, the map $N \rightarrow M$ so defined is a cofibration.

Definition 3.7 Let $A, B$ and $C$ be three topological spaces and $f: A \rightarrow B$ be a continuous map. The space of continuous maps $g: C \rightarrow B$ such that $g_{\mid A}=f$ is denoted by Top $f((C, A), B)$.

Lemma 3.8 [19] Let $M$ and $N$ be two $O$-infinitesimal bimodules. If $M$ is obtained from $N$ by attaching cells as in (6), then one has the homeomorphism

$$
\operatorname{Ibimod}_{O}^{g}((M, N) ; Y) \cong \operatorname{Coll}(S)^{g \circ f}((B, A) ; Y),
$$

with $f$ the attaching map and $g: N \rightarrow Y$ an $O$-infinitesimal bimodule map. 
Similarly, let $M$ and $N$ be two $O$-bimodules. If $M$ is obtained from $N$ by attaching cells as in (7), then one has the homeomorphism

$$
\operatorname{Bimod}_{O}^{g}((M, N) ; Y) \cong \operatorname{Coll}(S)^{g \circ f}((B, A) ; Y),
$$

with $f$ the attaching map and $g: N \rightarrow Y$ an $O$-bimodule map.

Definition 3.9 (i) As in [19] (see also [8, Lemma 4.24]), if $A$ and $B$ are $O-$ infinitesimal bimodules (resp. $O$-bimodules), and $A^{c}$ is a cofibrant replacement of $A$, then $\operatorname{Ibimod}_{O}\left(A^{c} ; B\right)$ (resp. $\left.\operatorname{Bimod}_{O}\left(A^{c} ; B\right)\right)$ is independent, up to weak equivalences, of the choice of a cofibrant replacement of $A$ since every $O$-infinitesimal bimodule (resp. $O$-bimodule) $B$ is fibrant. This space is called the space of derived $O$-infinitesimal bimodule (resp. $O$-bimodule) maps from $A$ to $B$ and is denoted by

$$
\operatorname{Ibimod}_{O}^{h}(A ; B) \quad\left(\text { resp. } \operatorname{Bimod}_{O}^{h}(A ; B)\right) .
$$

(ii) Similarly, Berger and Moerdijk define a model category structure on the category of $S$-colored operads in [2], and $\operatorname{Operad}_{S}^{h}(A ; B)$ denotes the space of derived $S$-operad maps from $A$ to $B$.

(iii) If $\mathcal{C}$ is the category $\operatorname{Bimod}_{\mathcal{A} t_{>0}}$ (resp. Operad $\{o ; c\}$ ), then for any cofibrant model $A$ of $\mathcal{A} c t_{>0}$, the family $A_{c}$ gives rise to a cofibrant replacement of $\mathrm{As}_{>0}$ in the category $\operatorname{Bimod}_{\mathrm{As}>0}$ (resp. Operad). As a consequence, the homotopy fiber of the projection onto the closed part is independent (up to weak equivalences) of the choice of a cofibrant model. By abuse of notation, we denote by

$$
\begin{aligned}
p_{1}^{h}: \operatorname{Bimod}_{\mathcal{A} c t>0}^{h}\left(\mathcal{A c t}_{>0} ; M\right) & \rightarrow \operatorname{Bimod}_{\mathrm{As}>0}^{h}\left(\operatorname{As}_{>0} ; M_{c}\right), \\
p_{2}^{h}: \operatorname{Operad}_{\{o ; c\}}^{h}\left(\mathcal{A c t}_{>0} ; O\right) & \rightarrow \operatorname{Operad}^{h}\left(\operatorname{As}_{>0} ; O_{c}\right),
\end{aligned}
$$

the projections onto the closed part whenever a cofibrant model of $\mathcal{A} t_{>0}$ is fixed. Furthermore, if the $\mathcal{A} c t_{>0}$-bimodule $M$ and the $\{o ; c\}$-operad $O$ are endowed with a map from $\mathcal{A} c t$, then all the spaces and maps are pointed. In this case, define

$$
\Omega\left(\operatorname{Bimod}_{\mathrm{As}>0}^{h}\left(\operatorname{As}_{>0} ; M_{c}\right) ; \operatorname{Bimod}_{\mathcal{A} c t>0}^{h}\left(\mathcal{A c t}_{>0} ; M\right)\right)
$$

and

$$
\Omega\left(\operatorname{Operad}^{h}\left(\operatorname{As}_{>0} ; O_{c}\right) ; \operatorname{Operad}_{\{o ; c\}}^{h}\left(\mathcal{A c t}_{>0} ; O\right)\right)
$$

to be respectively the homotopy fiber of the projection $p_{1}^{h}$ and $p_{2}^{h}$. They are called relative loop spaces.

Hence, in order to describe the spaces of derived maps and the relative loop spaces, we need to understand specific cofibrant replacement in the different categories involved. This is the aim of the two following subsections. 


\section{B Cofibrant replacement of $\mathcal{A c t}$ in $\operatorname{Ibimod}_{\mathcal{A} c t_{>0}}$}

Proposition 3.10 A cofibrant replacement of the $\mathcal{A} c t_{>0}$-infinitesimal bimodule $\mathcal{A} c t$ is the $\mathcal{A c t}_{>0}-$ infinitesimal bimodule $\boldsymbol{\Delta}$ given by

$$
\Delta(n ; c)=\Delta^{n} \quad \text { for } n \geq 0 \quad \text { and } \quad \Delta(n ; o)=\Delta^{n-1} \times[0,1] \text { for } n>0,
$$

where the structure is defined, for $1 \leq i \leq n$ (resp. $1 \leq i \leq n-1$ ) in the first (resp. second) map, by

$$
\begin{array}{lll}
-o^{i} *_{2 ; c}: \Delta(n ; c) \rightarrow \Delta(n+1 ; c), & \left(t_{1} ; \cdots ; t_{n}\right) & \mapsto\left(t_{1} ; \cdots ; t_{i} ; t_{i} ; \cdots ; t_{n}\right), \\
-o^{i} *_{2 ; c}: \Delta(n ; o) \rightarrow \Delta(n+1 ; o), & \left(t_{1} ; \cdots ; t_{n-1}\right) \times t \mapsto\left(t_{1} ; \cdots ; t_{i} ; t_{i} ; \cdots ; t_{n-1}\right) \times t, \\
-o^{n} *_{2 ; o}: \Delta(n ; o) \rightarrow \Delta(n+1 ; o), & \left(t_{1} ; \cdots ; t_{n-1}\right) \times t \mapsto\left(t_{1} ; \cdots ; t_{n-1} ; 1\right) \times t, \\
*_{2 ; c} \circ_{2}-: \Delta(n ; c) \rightarrow \Delta(n+1 ; c), & \left(t_{1} ; \cdots ; t_{n}\right) & \mapsto\left(0 ; t_{1} ; \cdots ; t_{n}\right), \\
*_{2 ; c} \circ_{1}-: \Delta(n ; c) \rightarrow \Delta(n+1 ; c), & \left(t_{1} ; \cdots ; t_{n}\right) & \mapsto\left(t_{1} ; \cdots ; t_{n} ; 1\right), \\
*_{2 ; o} \circ_{2}-: \Delta(n ; o) \rightarrow \Delta(n+1 ; o), & \left(t_{1} ; \cdots ; t_{n-1}\right) \times t \mapsto\left(0 ; t_{1} ; \cdots ; t_{n-1}\right) \times t, \\
*_{2 ; o} \circ_{1}-: \Delta(n ; c) \rightarrow \Delta(n+1 ; o), & \left(t_{1} ; \cdots ; t_{n}\right) & \mapsto\left(t_{1} ; \cdots ; t_{n}\right) \times 1 .
\end{array}
$$

Here, $\left(t_{1} ; \cdots ; t_{m}\right)$ is a nondecreasing sequence; that is, $t_{i} \leq t_{i+1}$ for $1 \leq i<m$.

Proof Since $\mathcal{A} c t_{>0}$ is generated as a colored operad by $*_{2 ; c}$ and $*_{2 ; o}$ with the relations (1) of Definition 1.4, the previous structure makes $\boldsymbol{\Delta}$ into an $\mathcal{A} c t_{>0}$-infinitesimal bimodule. Let $\boldsymbol{\Delta}_{N}$ be the sub- $\mathcal{A} c t_{>0}$-infinitesimal bimodule of $\boldsymbol{\Delta}$ generated by

$$
\{\boldsymbol{\Delta}(n ; c)\}_{n=0}^{N} \sqcup\{\boldsymbol{\Delta}(n ; o)\}_{n=1}^{N} \text { with } N \in \mathbb{N} .
$$

That is, $\boldsymbol{\Delta}_{N-1}(N ; c)$ is the boundary of the space $\boldsymbol{\Delta}(N ; c)$, whereas $\boldsymbol{\Delta}_{N-1}(N ; o)$ is composed of all the faces of dimension $N-1$ in $\Delta(N ; o)$ except $\Delta^{N-1} \times\{0\}$. By convention, $\boldsymbol{\Delta}_{-1}$ is the infinitesimal bimodule $\mathrm{Ib}_{\mathcal{A c t}>0}(\varnothing)$, and $\partial \Delta^{0}=\varnothing$. The space $\boldsymbol{\Delta}_{0}$ is obtained from $\boldsymbol{\Delta}_{-1}$ by the attaching cells

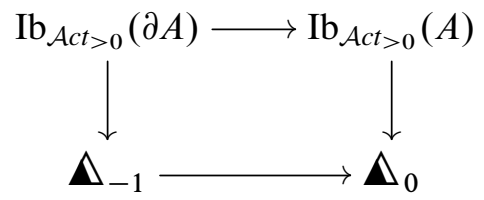

with $A(0 ; c)=\Delta^{0}$ and the empty set otherwise.

Let $B$ and $C$ be the $\{o ; c\}$-sequences given by $B(N ; o)=\Delta^{N-1} \times\{0\}, C(N ; o)=$ $\Delta^{N-1} \times[0,1], C(N ; c)=\Delta^{N}$ and the empty set otherwise. For $N \in \mathbb{N}_{>0}$, the 

$\Delta(1 ; c)$
$\Delta(2 ; c)$
$\Delta(3 ; c)$

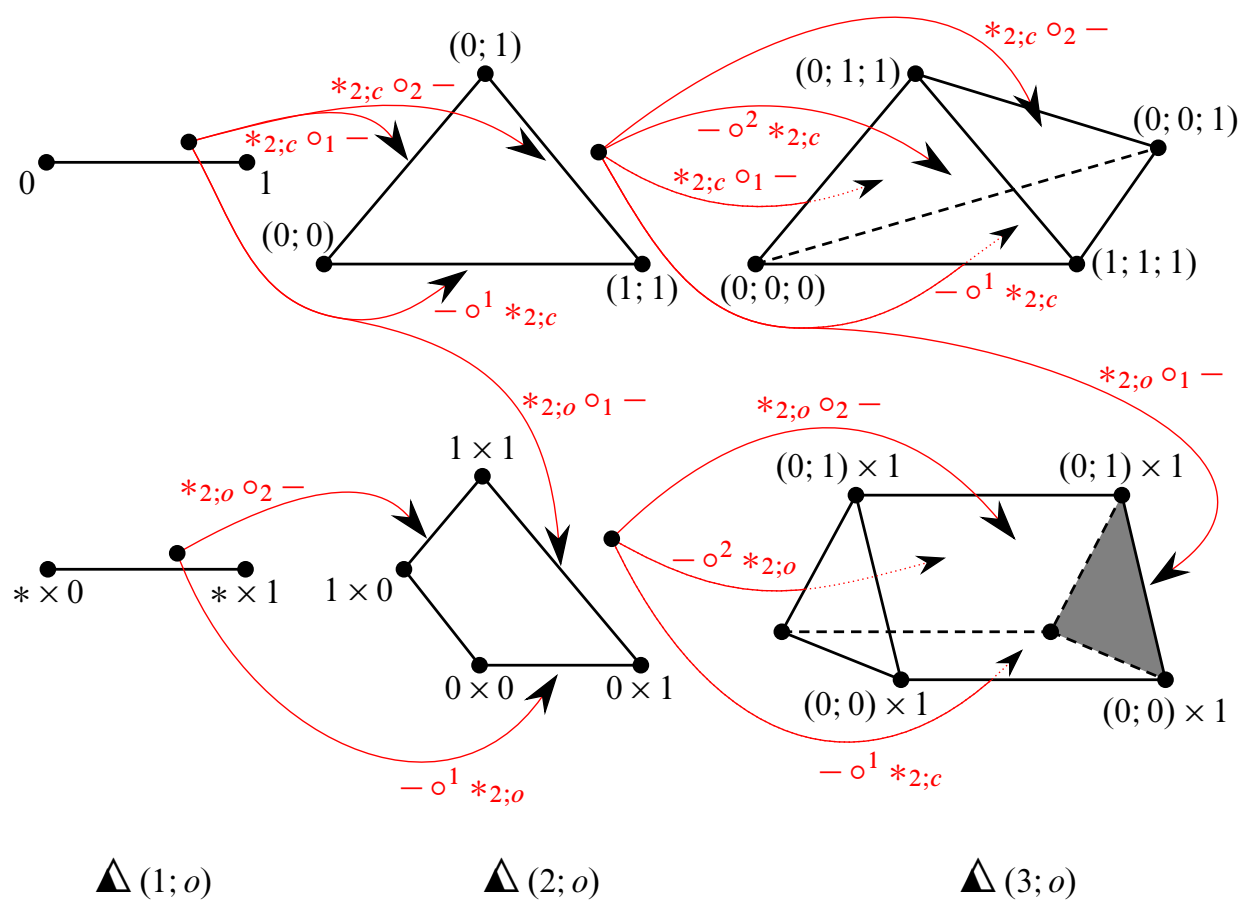

Figure 6: The structure of $\boldsymbol{\Delta}$.

infinitesimal bimodule $\boldsymbol{\Delta}_{N}$ is obtained from $\boldsymbol{\Delta}_{N-1}$ by the sequence of attaching cells:
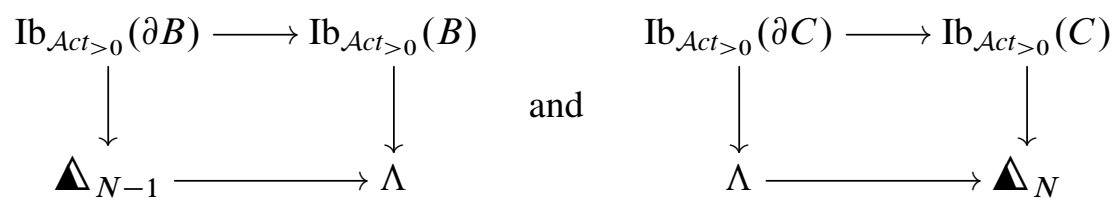

The attaching map $\partial B \rightarrow \boldsymbol{\Delta}_{N-1}$ is the restriction to the boundary of the map

$$
i: \Delta^{N-1} \rightarrow \Delta(N ; o), \quad\left(t_{1} ; \cdots ; t_{N-1}\right) \mapsto\left(t_{1} ; \cdots ; t_{N-1}\right) \times 0 .
$$

This step consists in adding the missing face of dimension $N-1$. The homeomorphisms $\boldsymbol{\Delta}_{N-1}(N ; o) \rightarrow \partial\left(\Delta^{N-1} \times[0,1]\right) \backslash \operatorname{Int}\left(\Delta^{N-1} \times\{0\}\right)$ and $B(N ; o) \rightarrow \Delta^{N-1} \times\{0\}$ give rise to a homeomorphism from $\Lambda(N ; o)$ to $\partial \boldsymbol{\Delta}(N ; o)=\partial C(N ; o)$, yielding the right hand side attaching map. For $N \geq n, \boldsymbol{\Delta}_{N}(n ; k)=\boldsymbol{\Delta}(n ; k)$ with $k \in\{o ; c\}$. Consequently, $\lim _{N} \boldsymbol{\Delta}_{N}=\boldsymbol{\Delta}$, and $\boldsymbol{\Delta}$ is cofibrant. The weak equivalence between $\boldsymbol{\Delta}$ and $\mathcal{A}$ ct is due to the convexity of $\boldsymbol{\Lambda}$ in each degree. 
Remark 3.11 According to Definition 3.9, the sequence given by $\Delta(n)=\boldsymbol{\Delta}(n ; c)=$ $\Delta^{n}$ inherits an $\mathrm{As}_{>0}$-infinitesimal bimodule structure, and it is a cofibrant replacement of As in the model category $\operatorname{Ibimod}_{\mathrm{As}_{>0}}$; see also [19, Proposition 3.2].

Theorem 3.12 Let $M$ be an $\mathcal{A c t}_{>0}$-infinitesimal bimodule. One has

$$
\operatorname{Ibimod}_{\mathcal{A} c t>0}^{h}(\mathcal{A} c t ; M) \simeq \operatorname{Ibimod}_{\mathrm{As}>0}^{h}\left(\mathrm{As} ; M_{c}\right) \simeq \operatorname{sTot}\left(M_{c}\right) .
$$

Proof From Proposition 3.10 and the previous remark, a cofibrant replacement of $\mathcal{A c t}$ in the model category $\operatorname{Ibimod}_{\mathcal{A} t>0}$ is given by $\boldsymbol{\Delta}$, and a cofibrant replacement of the associative operad As in the model category $\operatorname{Ibimod}_{\mathrm{As}>0}$ is given by $\Delta$. Since $M_{c}$ is an infinitesimal bimodule over $\mathrm{As}_{>0}$ (see Proposition 1.9), Definition 3.9 induces

$$
\operatorname{Ibimod}_{\mathrm{As}>0}^{h}\left(\mathrm{As} ; M_{c}\right) \simeq \operatorname{Ibimod}_{\mathrm{As}_{>0}}\left(\Delta ; M_{c}\right)
$$

and

$$
\operatorname{Ibimod}_{\mathcal{A} c t>0}^{h}(\mathcal{A c t} ; M) \simeq \operatorname{Ibimod}_{\mathcal{A} c t>0}(\boldsymbol{\Delta} ; M) .
$$

Let $i$ be the inclusion defined by

$$
i: \operatorname{Ibimod}_{\mathrm{As}_{>0}}\left(\Delta ; M_{c}\right) \hookrightarrow \operatorname{Ibimod}_{\mathcal{A c t}_{>0}}(\Delta ; M),
$$

which sends a point $f:=\left\{f_{n ; c}: \Delta^{n} \rightarrow M(n ; c)\right\}_{n \in \mathbb{N}}$ to the map $g$ defined by

$$
\begin{array}{ll}
g_{n ; c}: \Delta^{n} \rightarrow M(n ; c), & \left(t_{1} ; \cdots ; t_{n}\right) \mapsto f_{n ; c}\left(t_{1} ; \cdots ; t_{n}\right), \\
g_{n ; o}: \Delta^{n-1} \times I \rightarrow M(n ; o), & \left(t_{1} ; \cdots ; t_{n-1}\right) \times t \mapsto *_{2 ; o} \circ_{1} f_{n-1 ; c}\left(t_{1} ; \cdots ; t_{n-1}\right) .
\end{array}
$$

The space $\operatorname{Ibimod}_{\mathrm{As}>0}\left(\Delta ; M_{c}\right)$ is a deformation retract of $\operatorname{Ibimod}_{\mathcal{A c t}>0}(\boldsymbol{\Delta} ; M)$ with the homotopy

$$
H: \operatorname{Ibimod}_{\mathcal{A} c t>0}(\boldsymbol{\Delta} ; M) \times[0,1] \rightarrow \operatorname{Ibimod}_{\mathcal{A} c t>0}(\boldsymbol{\Delta} ; M)
$$

sending a point $(f \times u)$ to the map $H(f ; u)$ given by

$$
\begin{array}{ll}
H(f ; u)_{n ; c}: \Delta^{n} \rightarrow M(n ; c), & \left(t_{1} ; \cdots ; t_{n}\right) \mapsto f_{n ; c}\left(t_{1} ; \cdots ; t_{n}\right), \\
H(f ; u)_{n ; o}: \Delta^{n-1} \times I \rightarrow M(n ; o), & \left(t_{1} ; \cdots ; t_{n-1}\right) \times t \\
& \mapsto f_{n ; o}\left(\left(t_{1} ; \cdots ; t_{n-1}\right) \times(t u+(1-u))\right) .
\end{array}
$$

The map $H$ is continuous, and $H(f ; 1)=f$. Furthermore,

$$
\begin{aligned}
H(f ; 0)_{n ; o}\left(\left(t_{1} ; \cdots ; t_{n-1}\right) \times t\right) & =f_{n ; o}\left(\left(t_{1} ; \cdots ; t_{n-1}\right) \times 1\right) \\
& =f_{n ; o}\left(*_{2 ; o} \circ_{1}\left(t_{1} ; \cdots ; t_{n-1}\right)\right) \\
& =*_{2 ; o} \circ_{1} f_{n-1 ; c}\left(t_{1} ; \cdots ; t_{n-1}\right) .
\end{aligned}
$$


So $H(f ; 0)$ is in the image of the inclusion map $i$; also, for all $f \in \operatorname{Ibimod}_{\mathrm{As}>0}\left(\Delta ; M_{c}\right)$ and for all $u \in[0,1]$, we have $H(i(f) ; u)=i(f)$. Indeed,

$$
\begin{aligned}
(H(i(f) ; u))_{n ; o}\left(\left(t_{1} ; \cdots ; t_{n-1}\right) \times t\right) & =i(f)_{n ; o}\left(\left(t_{1} ; \cdots ; t_{n-1}\right) \times(t u+(1-u))\right) \\
& =\left(*_{2 ; o} \circ_{1} f_{n-1 ; c}\right)\left(t_{1} ; \cdots ; t_{n-1}\right) \\
& =i(f)_{n ; o}\left(\left(t_{1} ; \cdots ; t_{n-1}\right) \times t\right) .
\end{aligned}
$$

Corollary 3.13 Let $M$ denote an $\mathcal{A} t_{>0}$-bimodule such that $M(0 ; c) \simeq *$ and let $\eta: \mathcal{A c t} \rightarrow M$ be a map of $\mathcal{A} c t_{>0}$-bimodules. The following weak equivalences hold:

$\operatorname{Ibimod}_{\mathcal{A} t>0}^{h}(\mathcal{A} c t ; M) \simeq \operatorname{Ibimod}_{\mathrm{As}>0}^{h}\left(\mathrm{As} ; M_{c}\right) \simeq \Omega \operatorname{Bimod}_{\mathrm{As}>0}^{h}\left(\mathrm{As}_{>0} ; M_{c}\right)$.

Similarly, let $O$ denote an action-operad such that $O(0 ; c) \simeq O(1 ; c) \simeq *$. The following weak equivalences hold:

$\operatorname{Ibimod}_{\mathcal{A} c t>0}^{h}(\mathcal{A c t} ; O) \simeq \operatorname{Ibimod}_{\mathrm{As}>0}^{h}\left(\mathrm{As} ; O_{c}\right) \simeq \Omega^{2} \operatorname{Operad}_{\{o ; c\}}^{h}\left(\mathrm{As}_{>0} ; O_{c}\right)$.

Proof It is a consequence of Theorem 3.12 together with [19, Theorem 6.2] and [19, Theorem 7.2].

\section{C Cofibrant replacement of $\mathcal{A c t}_{>0}$ in $\operatorname{Bimod}_{\mathcal{A c t}>0}$}

Proposition 3.14 A cofibrant replacement of the $\mathcal{A c t}_{>0}$-bimodule $\mathcal{A c t}_{>0}$ is the $\mathcal{A c t}_{>0}$-bimodule $\square$ defined, for $n>0$, by

$$
\square(n ; c)=[0 ; 1]^{n-1} \text { and } \square(n ; o)=[0 ; 1]^{n-1},
$$

whose bimodule structure is given, for $1 \leq i \leq n$ (resp. $1 \leq i \leq n-1$ ) in the first (resp. second) map, by

$$
\begin{aligned}
-o^{i} *_{2 ; c}: \square(n ; c) \rightarrow \square(n+1 ; c), \quad\left(t_{1}, \ldots, t_{n-1}\right) \mapsto\left(t_{1}, \ldots, t_{i-1}, 0, t_{i}, \ldots, t_{n-1}\right), \\
-o^{i} *_{2 ; c}: \square(n ; o) \rightarrow \square(n+1 ; o), \quad\left(t_{1}, \ldots, t_{n-1}\right) \mapsto\left(t_{1}, \ldots, t_{i-1}, 0, t_{i}, \ldots, t_{n-1}\right), \\
-o^{n} *_{2 ; o}: \square(n ; o) \rightarrow \square(n+1 ; o), \quad\left(t_{1}, \ldots, t_{n-1}\right) \mapsto\left(t_{1}, \ldots, t_{n-1}, 0\right), \\
*_{2 ; c}(-;-): \square(n ; c) \times \square(m ; c) \rightarrow \square(n+m ; c), \\
\quad\left(t_{1}, \ldots, t_{n-1}\right) ;\left(t_{1}^{\prime}, \ldots, t_{m-1}^{\prime}\right) \mapsto\left(t_{1}, \ldots, t_{n-1}, 1, t_{1}^{\prime}, \ldots, t_{m-1}^{\prime}\right), \\
*_{2 ; o}(-;-): \square(n ; c) \times \square(m ; o) \rightarrow \square(n+m ; o), \\
\quad\left(t_{1}, \ldots, t_{n-1}\right) ;\left(t_{1}^{\prime}, \ldots, t_{m-1}^{\prime}\right) \mapsto\left(t_{1}, \ldots, t_{n-1}, 1, t_{1}^{\prime}, \ldots, t_{m-1}^{\prime}\right) .
\end{aligned}
$$

Proof Since $\mathcal{A} t_{>0}$ is generated as a colored operad by $*_{2 ; c}$ and $*_{2 ; o}$ with the relations (1) of Definition 1.4, the previous structure induces an $\mathcal{A} c t_{>0}$-bimodule structure on $\square$. For $N>0$ let $\square_{N}$ be the sub- $\mathcal{A c t}_{>0}$-bimodule of $\square$ generated 
by $\{\square(n ; k) \mid n \in\{1, \ldots, N\}, k \in\{o ; c\}\}$. In other words, the spaces $\square_{N-1}(n ; c)$ and $\square_{N-1}(n ; o)$ are the $(N-2)$-skeletons of $\square(n ; c)$ and $\square(n ; o)$, respectively. By convention, $\square_{0}$ is the $\mathcal{A c t}_{>0}$-bimodule $B_{\mathcal{A c t}>0}(\varnothing)$. The bimodule $\square_{N}$ is obtained from $\square_{N-1}$ by the attaching cells

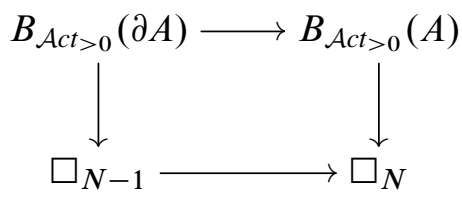

with $A$ the $\{o ; c\}$-sequence defined by $A(N ; c)=A(N ; o)=[0 ; 1]^{N-1}$ and the empty set otherwise.

For $N \geq n, \square_{N}(n ; k)=\square(n ; k)$ with $k \in\{o ; c\}$. Consequently, $\lim _{N} \square_{N}=\square$, and thus $\square$ is cofibrant. The weak equivalence between $\square$ and $\mathcal{A c t}_{>0}$ is due to the convexity of $\square$ in each degree.

Remark 3.15 According to Definition 3.9, the sequence given by $\square_{c}(n)=\square(n ; c)$ inherits an $\mathrm{As}_{>0}$-bimodule structure, and it is a cofibrant replacement of $\mathrm{As}_{>0}$ in the model category $\operatorname{Bimod}_{\mathrm{As}_{>0}}$; see [19, Proposition 4.1].

\section{Relative delooping of $\operatorname{sTot}\left(M_{o}\right)$}

Let $M$ be an $\mathcal{A c t}_{>0}$-bimodule endowed with a map $\eta: \mathcal{A} c t \rightarrow M$. Since the semi-

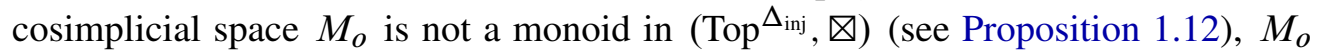
is not a bimodule over $\mathrm{As}_{>0}$, and we can not expect that its semitotalization has the homotopy type of a loop space. However, we will use the left module structure on $M_{o}$ to prove that the pair $\left(\operatorname{sTot}\left(M_{c}\right) ; \operatorname{sTot}\left(M_{o}\right)\right)$ has the homotopy type of an $\mathcal{S C}_{1}-$ space. The first step consists in showing that $\operatorname{sTot}\left(M_{o}\right)$ is weakly equivalent to the homotopy fiber of the map (8) of Definition 3.9. The next definition gives a model of this homotopy fiber using the cofibrant replacement $\square$ of $\mathcal{A c t}_{>0}$.

Definition 4.1 Let $\eta: \mathcal{A} c t \rightarrow M$ be an $\mathcal{A c t}_{>0}$-bimodule map, and let $\square \times I$ be the $\{o ; c\}$-sequence defined by

$(\square \times I)(n ; c)=\square(n ; c) \times[0,1] \quad$ and $\quad(\square \times I)(n ; o)=\square(n ; o) \times\{1\} \quad$ for $n>0$.

A relative loop in $M$ is an $\{o ; c\}$-sequence map $g$ from $\square \times I$ to $M$ defined by $g_{n ; c}: \square(n ; c) \times[0,1] \rightarrow M(n ; c) \quad$ and $\quad g_{n ; o}: \square(n ; o) \times\{1\} \rightarrow M(n ; o)$ for $n>0$, 
satisfying

$$
\begin{array}{ll}
g_{n ; c}\left(x \circ^{i} *_{2 ; c} ; t\right)=g_{n-1 ; c}(x ; t) \circ^{i} *_{2 ; c} & \text { for } x \in \square(n-1 ; c) \text { and } 1 \leq i \leq n-1, \\
g_{n ; c}\left(*_{2 ; c}(x ; y) ; t\right) & \\
\quad=*_{2 ; c}\left(g_{l ; c}(x ; t) ; g_{n-l ; c}(y ; t)\right) & \text { for } x \in \square(l ; c) \text { and } y \in \square(n-l ; c), \\
g_{n ; o}\left(x \circ^{i} *_{2 ; c} ; 1\right)=g_{n-1 ; o}(x ; 1) \circ^{i} *_{2 ; c} & \text { for } x \in \square(n-1 ; o) \text { and } 1 \leq i \leq n-2, \\
g_{n ; o}\left(x \circ^{n-1} *_{2 ; o} ; 1\right)=g_{n-1 ; o}(x ; 1) \circ^{n-1} *_{2 ; o} & \text { for } x \in \square(n-1 ; o), \\
g_{n ; o}(* 2 ; o & \\
\quad=*_{2 ; o}\left(g_{l ; c}(x ; 1) ; g_{n-l ; o}(y ; 1)\right) & \text { for } x \in \square(l ; c) \text { and } y \in \square(n-l ; o),
\end{array}
$$

with the boundary conditions $g_{n ; c}(x ; 0)=\eta\left(*_{n ; c}\right)$ for $x \in \square(n ; c)$. This model for the space of relative loops is denoted by $\Omega\left(\operatorname{Bimod}_{\mathrm{As}_{>0}}\left(\square_{c} ; M_{c}\right) ; \operatorname{Bimod}_{\mathcal{A c t}_{>0}}(\square ; M)\right)$.

Theorem 4.2 If $M$ is an $\mathcal{A} c t_{>0}$-bimodule endowed with a map of $\mathcal{A} c t_{>0}$-bimodules $\eta: \mathcal{A c t} \rightarrow M$, then

$$
\operatorname{sTot}\left(M_{o}\right) \simeq \Omega\left(\operatorname{Bimod}_{\mathrm{As}>0}^{h}\left(\mathrm{As}_{>0} ; M_{c}\right) ; \operatorname{Bimod}_{\mathcal{A} c t_{>0}}^{h}\left(\mathcal{A c t}_{>0} ; M\right)\right) .
$$

Proof It is a consequence of Proposition 4.4 and Proposition 4.5.

Notation 4.3 Let $M$ be an $\mathcal{A} c t_{>0}$-bimodule endowed with a map $\eta$ : $\mathcal{A} c t \rightarrow M$. The $\{o ; c\}$-sequence $M^{*}$ given by

$$
M^{*}(n ; c)=\eta\left(*_{n ; c}\right) \quad \text { for } n \geq 0, \quad M^{*}(n ; o)=M(n ; o) \quad \text { for } n>0,
$$

and the empty set otherwise, inherits from $M$ an $\mathcal{A} c t_{>0}$-bimodule structure with a $\operatorname{map} \eta: \mathcal{A c t} \rightarrow M^{*}$.

Proposition 4.4 We have the following weak equivalence:

$$
\operatorname{sTot}\left(M_{o}\right) \simeq \operatorname{Bimod}_{\mathcal{A} c t_{>0}}^{h}\left(\mathcal{A} c t_{>0} ; M^{*}\right)
$$

Proof As seen in the first section, $\operatorname{sTot}\left(M_{o}\right) \simeq \operatorname{Ibimod}_{\mathrm{As}>0}^{h}\left(\mathrm{As} ; M_{o}\right)$ using the structure (2). The first step of the proof consists in building an alternative cofibrant replacement $\tilde{\square}$ of As in the category of infinitesimal bimodules over As $>0$ such that there exists a map $\xi: \operatorname{Bimod}_{\mathcal{A} c t>0}\left(\square ; M^{*}\right) \rightarrow \operatorname{Ibimod}_{\mathrm{As}>0}\left(\tilde{\square} ; M_{o}\right)$. Let us recall that a point $g \in \operatorname{Bimod}_{\mathcal{A} c t>0}\left(\square ; M^{*}\right)$ is described by

$$
\begin{array}{ll}
g_{n ; c}: \square(n ; c) \rightarrow M^{*}(n ; c), \quad x \mapsto \eta\left(*_{n ; c}\right) & \text { for } n>0, \\
g_{n ; o}: \square(n ; o) \rightarrow M^{*}(n ; o) & \text { for } n>0,
\end{array}
$$


satisfying

$$
\begin{aligned}
g_{n ; o}\left(x \circ^{i} *_{2 ; c}\right) & =g_{n-1 ; o}(x) \circ^{i} *_{2 ; c} & & \text { for } x \in \square(n-1 ; o) \text { and } i \neq n-1, \\
g_{n ; o}\left(x \circ^{n-1} *_{2 ; o}\right) & =g_{n-1 ; o}(x) \circ^{n-1} *_{2 ; o} & & \text { for } x \in \square(n-1 ; o), \\
g_{n ; o}\left(*_{2 ; o}(x ; y)\right) & =*_{2 ; o}\left(g_{l ; c}(x) ; g_{n-l ; o}(y)\right) & & \\
& =*_{2 ; o}\left(\eta(* l ; c) ; g_{n-l ; o}(y)\right) & & \text { for } x \in \square(l ; c) \text { and } y \in \square(n-l ; o) .
\end{aligned}
$$

Define $\sim$ to be the equivalence relation on $[0,1]^{n}$ generated by

such that

$$
\left(t_{1}, \ldots, t_{n}\right) \sim\left(t_{1}^{\prime}, \ldots, t_{n}^{\prime}\right) \quad \Longleftrightarrow \quad \text { there exists } i \in\{1, \ldots, n\}
$$

$$
t_{i}=t_{i}^{\prime}=1 \quad \text { and } \quad t_{j}=t_{j}^{\prime} \quad \text { for } j>i \text {. }
$$

We denote by $\tilde{\square}$ the sequence $\left\{\tilde{\square}(n)=[0,1]^{n} / \sim\right\}_{n \geq 0}$.

The map $g$ induces a sequence map $\widetilde{g}:=\left\{\widetilde{g}_{n+1}: \tilde{\square}(n) \rightarrow M^{*}(n+1 ; o)=M_{o}^{n}\right\}_{n \geq 0}$. Indeed, if $\left(t_{1}, \ldots, t_{n}\right) \sim\left(t_{1}^{\prime}, \ldots, t_{n}^{\prime}\right)$, then there exists $i$ such that $t_{i}=t_{i}^{\prime}=1$ and $t_{j}=t_{j}^{\prime}$ for $j>i$. So the following equalities hold:

$$
\begin{aligned}
g_{n+1 ; o}\left(t_{1}, \ldots, t_{n}\right) & =g_{n+1 ; o}\left(t_{1}, \ldots, t_{i-1}, 1, t_{i+1}, \ldots, t_{n}\right) \\
& =g_{n+1 ; o}\left(*_{2 ; o}\left(\left(t_{1}, \ldots, t_{i-1}\right) ;\left(t_{i+1}, \ldots, t_{n}\right)\right)\right) \\
& =*_{2 ; o}\left(g_{i ; c}\left(t_{1}, \ldots, t_{i-1}\right) ; g_{n-i ; o}\left(t_{i+1}, \ldots, t_{n}\right)\right) \\
& =g_{n+1 ; o}\left(t_{1}^{\prime}, \ldots, t_{n}^{\prime}\right) .
\end{aligned}
$$

This equivalence relation is the usual way to describe a simplex as a quotient of the cube. So there exists an homeomorphism between $\tilde{\square}(n)$ and $\Delta^{n}$. For this work we will just prove that $\tilde{\square}$ is a cofibrant replacement of As as an $\mathrm{As}_{>0}$-infinitesimal bimodule. The infinitesimal bimodule structure is given, for $1 \leq i \leq n$, by
(i) $-\circ^{i} *_{2}: \tilde{\square}(n) \rightarrow \tilde{\square}(n+1)$,
$\left(t_{1}, \ldots, t_{n}\right) \mapsto\left(t_{1}, \ldots, t_{i-1}, 0, t_{i}, \ldots, t_{n}\right)$,
(ii) $*_{2} \circ_{1}-: \tilde{\square}(n) \rightarrow \tilde{\square}(n+1)$,
$\left(t_{1}, \ldots, t_{n}\right) \mapsto\left(t_{1}, \ldots, t_{n}, 0\right)$,
(iii) $*_{2} \circ_{2}-: \tilde{\square}(n) \rightarrow \tilde{\square}(n+1)$,
$\left(t_{1}, \ldots, t_{n}\right) \mapsto\left(1, t_{1}, \ldots, t_{n}\right)$.

This structure satisfies the infinitesimal bimodule axioms over $\mathrm{As}_{>0}$, and it makes $\tilde{g}$ into an $\mathrm{As}_{>0}$-infinitesimal bimodule map. Furthermore, $\tilde{\square}$ is a cofibrant replacement of the $\mathrm{As}_{>0}$-infinitesimal bimodule As, as we will now show.

Cofibrant Let $\tilde{\square}_{n}$ be the sub-As $>0$-infinitesimal bimodule of $\tilde{\square}$ generated by $\{\tilde{\square}(i) \mid 0 \leq i \leq n\}$ for $n \in \mathbb{N}$. By convention, $\tilde{\square}_{-1}$ is the As $>0$-infinitesimal bimodule $\mathrm{Ib}_{\mathrm{As}>0}(\varnothing)$. Let us notice that the boundary of $\tilde{\square}(n)$ is determined by $\tilde{\square}(n-1)$ and 
its infinitesimal bimodule structure. Indeed, the map $[0,1]^{n} \rightarrow \tilde{\square}(n)$ preserves the boundary, and by definition, a point in $\partial[0,1]^{n}$ has one of the following forms:

$$
\left(t_{1}, \ldots, t_{l-1}, 0, t_{l+1}, \ldots, t_{n}\right) \text { or }\left(t_{1}, \ldots, t_{l-1}, 1, t_{l+1}, \ldots, t_{n}\right) .
$$

In the first case, the class of such a point lies in $\tilde{\square}_{n-1}$ by the axioms (i) and (ii). In the second case, we have the following identification:

$$
\begin{aligned}
{\left[\left(t_{1}, \ldots, t_{l-1}, 1, t_{l+1}, \ldots, t_{n}\right)\right] } & =\left[(\underbrace{1, \ldots, 1}_{l}, t_{l+1}, \ldots, t_{n})\right] \\
& =*_{2} \circ_{2}\left[(\underbrace{1, \ldots, 1}_{l-1}, t_{l+1}, \ldots, t_{n})\right] .
\end{aligned}
$$

Consequently, $\tilde{\square}_{n}$ is obtained from $\tilde{\square}_{n-1}$ by the pushout diagram

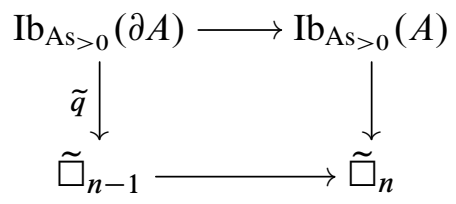

where $A$ is the sequence given by $A(n)=[0,1]^{n}$ and the empty set otherwise. The attaching map is the restriction of the quotient map $q:[0,1]^{n} \rightarrow[0,1]^{n} / \sim$ to the boundary. Moreover, if $n \geq i$, then $\tilde{\square}_{n}(i)=\tilde{\square}(i)$, and the map $\partial A \rightarrow A$ is a cofibration. So $\lim _{n} \tilde{\square}_{n}=\tilde{\square}$, and thus $\tilde{\square}$ is cofibrant.

This construction implies that $\tilde{\square}(m)$ is a CW-complex. We recall that if $A(n)=[0,1]^{n}$ and the empty set otherwise, then the points in $\operatorname{Ib}_{\mathrm{As}>0}(A)(m)$ are the pairs $(t ; x)$ with $x \in A(n)$ and $t$ a $\{c\}$-tree satisfying the following conditions:

$$
\left\{\begin{array}{l}
t \text { has } m \text { leaves, } \\
\text { for all } v \in V(t) \backslash\{p\}, \text { we have }|v|>1, \text { and } \\
|p|=n
\end{array}\right.
$$

We denote by $\operatorname{tr}_{m}^{n}$ the number of $\{c\}$-trees satisfying the conditions in (11). The space $\tilde{\square}_{0}(m)$ is the disjoint union of $\operatorname{tr}_{m}^{0}$ points, that is, a CW-complex. Assume $\tilde{\square}_{n-1}(m)$ is a CW-complex for all $m \geq 0$. For $m \leq n-1$, we have that $\tilde{\square}_{n}(m)=\tilde{\square}_{n-1}(m)=\tilde{\square}(m)$ is a CW-complex. The pushout (10) implies that $\tilde{\square}_{n}(n)=\tilde{\square}(n)$ is a CW-complex. Finally, for $m>n$, the space $\tilde{\square}_{n}(m)$ is obtained from the CW-complex $\tilde{\square}_{n-1}(m)$ by attaching $\operatorname{tr}_{m}^{n}$ cells of dimension $n$ according to the infinitesimal bimodule structure over $\mathrm{As}_{>0}$, and is thus a CW-complex.

Contractible The map $q:[0,1]^{n} \rightarrow \widetilde{\square}(n)$ is a continuous map between compact $\mathrm{CW}$-complexes. Since the fiber of $q$ over a point $\left(t_{1}, \ldots, t_{i-1}, 1, t_{i+1}, \ldots, t_{n}\right)$, with 
$t_{j} \neq 1$ for $j>i$, is homeomorphic to the contractible space $[0,1]^{i-1}$, the map $q$ is a weak equivalence [18, Main Theorem]. Hence $\tilde{\square}(n)$ is contractible.

Since $\tilde{\square}$ is a cofibrant replacement of As as an infinitesimal bimodule over $\mathrm{As}_{>0}$, the semitotalization $\operatorname{sTot}\left(M_{o}\right)$ is weakly equivalent to $\operatorname{Ibimod}_{\mathrm{As}>0}\left(\tilde{\square} ; M_{o}\right)$, and we have a map

$$
\xi: \operatorname{Bimod}_{A c t>0}\left(\square ; M^{*}\right) \rightarrow \operatorname{Ibimod}_{\mathrm{As}_{>0}}\left(\tilde{\square} ; M_{o}\right), \quad g \mapsto \widetilde{g} .
$$

In order to prove that $\xi$ is a weak equivalence, we will introduce two towers of fibrations. For $k \geq 0$, define $A_{k}$ and $B_{k}$ to be the subspaces

$$
A_{k} \subset \underbrace{\prod_{i=1}^{k+1} \operatorname{Top}\left(\square(i ; c) ; M^{*}(i ; c)\right)}_{\text {reduced to a point }} \times \prod_{i=1}^{k+1} \operatorname{Top}\left(\square(i ; o) ; M^{*}(i ; o)\right)
$$

and

$$
B_{k} \subset \prod_{i=0}^{k} \operatorname{Top}\left(\tilde{\square}(i) ; M_{o}^{i}\right),
$$

with $A_{k}$ satisfying the $\mathcal{A} t_{>0}$-bimodule relations and $B_{k}$ the $\mathrm{As}_{>0}$-infinitesimal bimodule relations. In other words, $A_{k}$ and $B_{k}$ are the spaces $\operatorname{Bimod}_{\mathcal{A c t}>0}\left(\square_{k+1} ; M^{*}\right)$ and $\operatorname{Ibimod}_{\mathrm{As}>0}\left(\tilde{\square}_{k} ; M_{o}\right)$, respectively, where $\square_{k+1}$ is the sub-Act $t_{>0}$-bimodule introduced in the proof of Proposition 3.14. The projection

$$
\prod_{i=1}^{k+1} \operatorname{Top}\left(\widetilde{\square}(i) ; M_{o}^{i}\right) \rightarrow \prod_{i=1}^{k} \operatorname{Top}\left(\widetilde{\square}(i) ; M_{o}^{i}\right)
$$

induces a map $B_{k+1} \rightarrow B_{k}$. From Lemma 3.3, the following map is a fibration:

$$
\operatorname{Top}\left(\tilde{\square}(k+1) ; M_{o}^{k+1}\right) \rightarrow \operatorname{Top}\left(\partial \tilde{\square}(k+1) ; M_{o}^{k+1}\right) .
$$

The space $B_{k+1}$ is obtained from $B_{k}$ by the following pullback diagram:

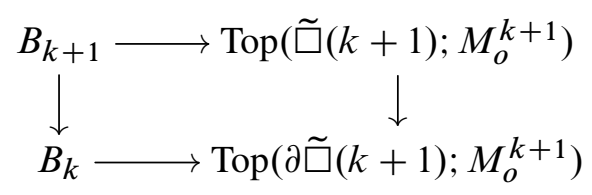

Since the fibrations are preserved by pullbacks, $B_{k+1} \rightarrow B_{k}$ is a fibration. Similarly, the next pullback square makes the map $A_{k+1} \rightarrow A_{k}$ induced by the projection into 
a fibration:

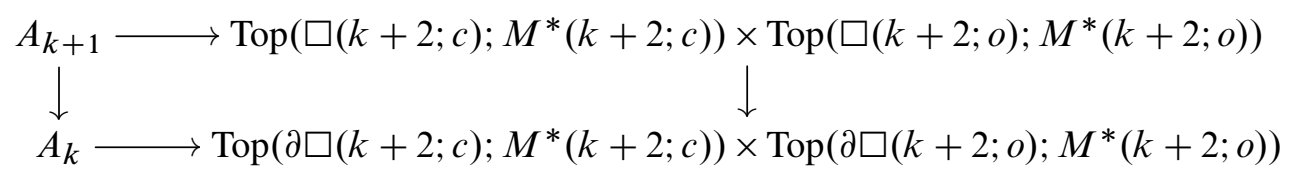

So we consider the two towers of fibrations

$$
\begin{aligned}
& A_{0} \longleftarrow A_{1} \longleftarrow \cdots \longleftarrow A_{k} \longleftarrow A_{k+1} \longleftarrow \cdots \longleftarrow \\
& B_{0} \longleftarrow B_{1} \longleftarrow \cdots \longleftarrow B_{k} \longleftarrow B_{k+1} \longleftarrow \cdots \longleftarrow
\end{aligned}
$$

such that

$$
\begin{aligned}
& A_{\infty}=\lim _{k} A_{k} \simeq \operatorname{holim}_{k} A_{k} \simeq \operatorname{Bimod}_{\mathcal{A c t}>0}\left(\square ; M^{*}\right), \\
& B_{\infty}=\lim _{k} B_{k} \simeq \operatorname{holim}_{k} B_{k} \simeq \operatorname{Ibimod}_{\mathrm{As}>0}\left(\tilde{\square} ; M_{o}\right) .
\end{aligned}
$$

By restriction, the map $\xi$ induces a map between the two towers

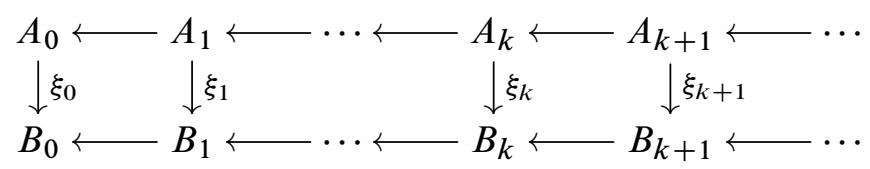

with $\xi=\lim _{k} \xi_{k}=\operatorname{holim}_{k} \xi_{k}$. Consequently, $\xi$ is a weak equivalence if each $\xi_{k}$ is a weak equivalence. We will prove this result by induction on $k$.

First, note that $\xi_{0}$ and $\xi_{1}$ coincide with the identity. They are weak equivalences. Now assume that $\xi_{k-1}$ is a weak equivalence. We consider the following diagram, where $g$ is a point in $A_{k-1}, F_{A}$ is the fiber over $g$ and $F_{B}$ the fiber over $\xi_{k-1}(g)$ :

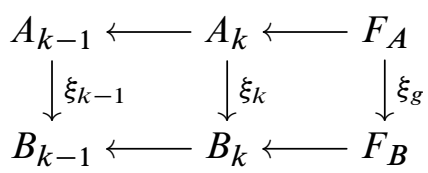

Since the two left horizontal arrows are fibrations, the map $\xi_{k}$ is a weak equivalence if the induced map $\xi_{g}$ is a weak equivalence.

The fiber $F_{A}$ is homeomorphic to the space $\operatorname{Top}^{g_{k+1 ; o}}\left(\left([0,1]^{k} ; \partial[0,1]^{k}\right) ; M(k+1 ; o)\right)$ by Lemma 3.8. Similarly, $\widetilde{\square}_{k}$ is obtained from $\tilde{\square}_{k-1}$ by the pushout diagram (10). So the fiber $F_{B}$ is homeomorphic to Top ${ }^{\xi_{k-1}(g)_{k} \circ q}\left(\left([0,1]^{k} ; \partial[0,1]^{k}\right) ; M(k+1 ; o)\right)$, and we have the following commutative square:

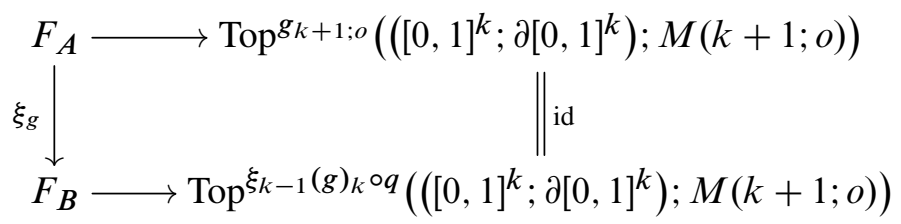


Consequently, $\xi_{k}$ is a weak equivalence.

Proposition 4.5 The space $\Omega\left(\operatorname{Bimod}_{\mathrm{As}_{>0}}^{h}\left(\operatorname{As}_{>0} ; M_{c}\right) ; \operatorname{Bimod}_{\mathcal{A c t}_{>0}}^{h}\left(\mathcal{A c t}_{>0} ; M\right)\right)$ is weakly equivalent to the space $\operatorname{Bimod}_{\mathcal{A c t}_{>0}}^{h}\left(\mathcal{A}_{c t}>0 ; M^{*}\right)$.

Proof In this proof, $\square$ will serve as a cofibrant model of the $\mathcal{A c t}_{>0}$-bimodule $\mathcal{A c t}_{>0}$. Through the following inclusion, we can consider $\operatorname{Bimod}_{\mathcal{A} c t>0}\left(\square ; M^{*}\right)$ as a subspace of $\Omega\left(\operatorname{Bimod}_{\mathrm{As}_{>0}}\left(\square_{c} ; M_{c}\right) ; \operatorname{Bimod}_{\mathcal{A c t}>0}(\square ; M)\right)$ :

$i: \operatorname{Bimod}_{\mathcal{A} c t>0}\left(\square ; M^{*}\right) \rightarrow \Omega\left(\operatorname{Bimod}_{\mathrm{As}_{>0}}\left(\square_{c} ; M_{c}\right) ; \operatorname{Bimod}_{\mathcal{A}_{>}>0}(\square ; M)\right)$,

$$
g \mapsto\left\{\begin{array}{l}
\widetilde{g}_{n ; c}: \square(n ; c) \times[0,1] \rightarrow M(n ; c) ;(x ; t) \mapsto \eta\left(*_{n ; c}\right), \\
\widetilde{g}_{n ; o}: \square(n ; o) \times\{1\} \rightarrow M(n ; o) ;(x ; 1) \mapsto g_{n ; o}(x) .
\end{array}\right.
$$

In order to show that $i$ is a weak equivalence, we introduce two towers of fibrations. One of them is the tower $A_{k}$ of Proposition 4.4. The second one is defined by

$$
C_{k} \subset \prod_{i=1}^{k+1} \operatorname{Top}(\square(i ; c) \times[0,1] ; M(i ; c)) \times \prod_{i=1}^{k+1} \operatorname{Top}(\square(i ; o) ; M(i ; o)),
$$

satisfying the relations of Definition 4.1. The map $C_{k+1} \rightarrow C_{k}$ induced by the projection is a fibration due to Lemma 3.3 and the following pullback diagram:

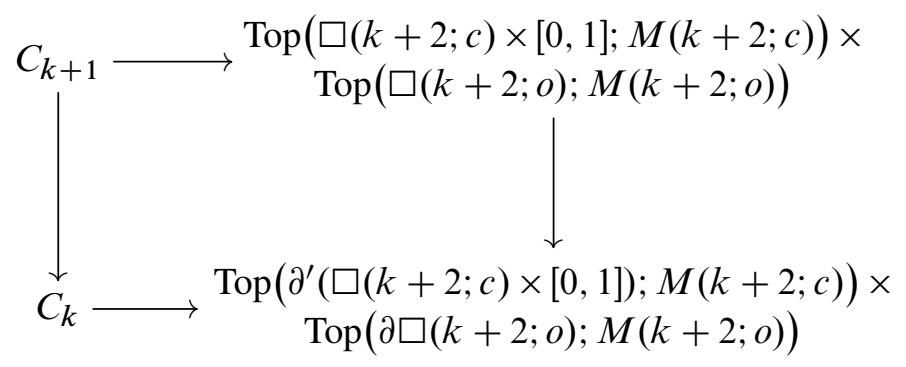

Here,

$$
\partial^{\prime}(\square(k+2 ; c) \times[0,1])=\square(k+2 ; c) \times\{0\} \cup \partial \square(k+2 ; c) \times[0,1] .
$$

The restriction of the inclusion $i$ induces a map between the two towers:

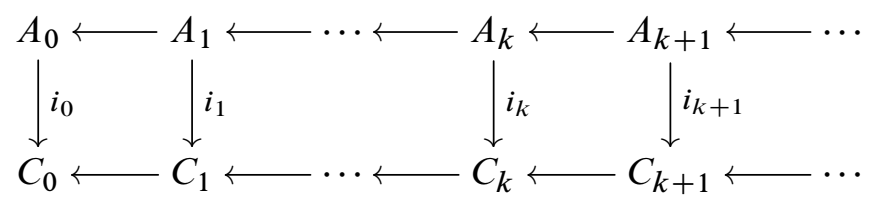


We will prove that $i$ is a weak equivalence by induction on $k$. If $k=0$, a point in $C_{0}$ is a pair $\left(g_{1 ; c} ; g_{1 ; o}\right)$, and the points in the image of $i_{0}$ are the pairs satisfying

$$
g_{1 ; c}: \square(1 ; c) \times[0,1] \rightarrow M(1 ; c), \quad(* ; t) \mapsto \eta\left(*_{1 ; c}\right) .
$$

Since $g_{1 ; c}(* ; 0)=\eta\left(*_{1 ; c}\right)$ for any pair in $C_{0}$, the inclusion $i_{0}$ induces the following deformation retract:

$H: C_{0} \times[0,1] \rightarrow C_{0}, \quad y=\left(\left(g_{1 ; c} ; g_{1 ; o}\right) ; t_{1}\right) \mapsto\left\{\begin{array}{l}H(y)_{1 ; c}(* ; t)=g_{1 ; c}\left(* ; t\left(1-t_{1}\right)\right), \\ H(y)_{1 ; o}(* ; 1)=g_{1 ; o}(* ; 1) .\end{array}\right.$

From now on, we assume that $i_{k-1}$ is a weak equivalence for $k \geq 1$. We consider the following diagram, where $g$ is a point in $A_{k-1}, F_{A}$ is the fiber over $g$ and $F_{C}$ the fiber over $i_{k-1}(g)$ :

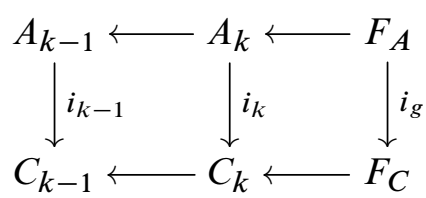

Since the two left horizontal arrows are fibrations, the map $i_{k}$ is a weak equivalence if the induced map $i_{g}$ is a weak equivalence.

A point in $F_{C}$ is defined by a pair $\left(g_{k+1 ; c} ; g_{k+1 ; o}\right)$ satisfying the relations seen in Definition 4.1. Since $g_{k+1 ; c}$ is in the fiber over $i_{k-1}(g)$, the map sends all the faces of $\square(k+1 ; c) \times[0,1]$ to $\eta\left(*_{k+1 ; c}\right)$, except for the face $\square(k+1 ; c) \times\{1\}$. Furthermore, they are no interaction between $g_{k+1 ; c}$ and $g_{k+1 ; o}$.

On the other hand, the points in the image of $i_{g}$ coincide with the pair $\left(g_{k+1 ; c} ; g_{k+1 ; o}\right)$ such that

$$
g_{k+1 ; c}: \square(k+1 ; c) \times[0,1] \rightarrow M(k+1 ; c), \quad(x ; t) \mapsto \eta\left(*_{k+1 ; c}\right) .
$$

In order to prove that $i_{g}$ induces a deformation retract, we introduce the homotopy (also described in [11, Proposition 0.16])

$$
H:(\square(k+1 ; c) \times[0,1]) \times[0,1] \rightarrow \square(k+1 ; c) \times[0,1]
$$

illustrated by the following picture:

In other words, the points in the image of $i_{g}$ coincide with the pairs such that

$$
g_{k+1 ; c}(x ; t)=g_{k+1 ; c}(H((x ; t) ; 1)) \quad \text { for } x \in \square(k+1 ; c) \text { and } t \in[0,1] .
$$

Finally, the deformation retract $H_{2}: F_{C} \times[0,1] \rightarrow F_{C}$ is given by

$$
\begin{aligned}
y & =\left(\left(g_{k+1 ; c} ; g_{k+1 ; o}\right) ; t_{1}\right) \\
& \mapsto \begin{cases}H_{2}(y)_{k+1 ; c}(x ; t)=g_{k+1 ; c}\left(H\left((x ; t) ; t_{1}\right)\right) & \text { for } x \in \square(k+1 ; c) \text { and } t \in[0,1], \\
H_{2}(y)_{k+1 ; o}(x ; 1)=g_{k+1 ; o}(x ; 1) & \text { for } x \in \square(k+1 ; o) .\end{cases}
\end{aligned}
$$

Thus, the space $\Omega\left(\operatorname{Bimod}_{\mathrm{As}>0}\left(\square_{c} ; M_{c}\right)\right.$; $\left.\operatorname{Bimod}_{\mathcal{A} t_{>0}}(\square ; M)\right)$ is weakly equivalent to $\operatorname{Bimod}_{\mathcal{A c t}>0}\left(\square ; M^{*}\right)$.

\section{Double relative delooping: a particular case}

First of all we recall that, for any pointed continuous map $f: A \rightarrow X$, the homotopy fiber hofib $(f)$ and the loop space $\Omega X$ based at $*$ are weakly equivalent to the pullback diagrams (I) and (II):
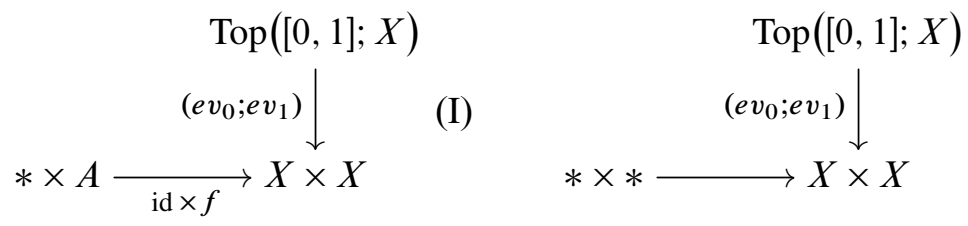

By the double relative loop space $\Omega^{2}(X ; A)$, we mean the loop space of the homotopy fiber hofib $(f)$. Since finite colimits commute, the double loop space can also be defined by the homotopy fiber of the continuous map $\Omega f$.

From now on, let $O$ be a multiplicative operad; that is, there exists an operad map $\alpha$ : As $\rightarrow O$. Let $B$ be an $O$-bimodule equipped with an $O$-bimodule map $\beta: O \rightarrow B$. If we assume that $B(0) \simeq *$, we know from [19, Theorem 6.2] and the $\mathrm{As}_{>0}-$ bimodule map $\beta \circ \alpha$ : As $\rightarrow B$ that $\operatorname{sTot}(B)$ is weakly equivalent to the loop space $\Omega \operatorname{Bimod}_{\mathrm{As}>0}^{h}\left(\mathrm{As}_{>0} ; B\right)$. Since $B$ is not an operad, we can not expect that its semitotalization has the homotopy type of a double loop space. However, we will prove that $\operatorname{Bimod}_{\mathrm{As}_{>0}}^{h}\left(\mathrm{As}_{>0} ; B\right)$ has the homotopy type of a relative loop space by building an $\{o ; c\}$-operad $X$ from the pair $(O ; B)$ as follows:

$$
X(n ; c)=O(n) \quad \text { for } n \geq 0, \quad X(n ; o)=B(n-1) \quad \text { for } n>0,
$$


and the empty set otherwise. The operadic structure is defined by

$$
\begin{array}{ll}
\circ_{i}: X(n ; c) \times X(m ; c) \rightarrow X(n+m-1 ; c), & (x ; y) \mapsto x \circ_{i} y, \\
\circ_{i}: X(n ; o) \times X(m ; c) \rightarrow X(n+m-1 ; o), & (x ; y) \mapsto x \circ^{i} y, \\
\circ_{n}: X(n ; o) \times X(m ; o) \rightarrow X(n+m-1 ; o), & (x ; y) \mapsto \alpha\left(*_{2}\right)(x ; y),
\end{array}
$$

using the operadic structure of $O$, the right $O$-bimodule structure of $B$, and the left $O$-bimodule structure of $B$, respectively. The operadic axioms are satisfied except the unit axiom. This axiom holds under the assumption

$$
\alpha\left(*_{2}\right)\left(\beta \circ \alpha\left(*_{0}\right) ; x\right)=\alpha\left(*_{2}\right)\left(x ; \beta \circ \alpha\left(*_{0}\right)\right)=x \quad \text { for } x \in X(m ; o) .
$$

Under this assumption, $X$ is an action-operad with

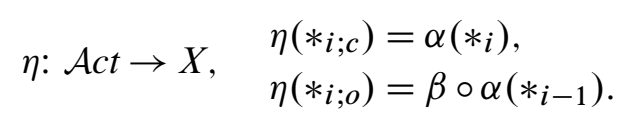

Theorem 5.1 Under assumption (13), $\operatorname{Bimod}_{\mathrm{As}>0}^{h}\left(\mathrm{As}_{>0} ; B\right)$ is weakly equivalent to the relative loop space $\Omega\left(\operatorname{Operad}^{h}\left(\operatorname{As}_{>0} ; O\right) ; \operatorname{Operad}_{\{o ; c\}}^{h}\left(\mathcal{A c t}_{>0} ; X\right)\right)$.

Proof It is a consequence of Propositions 5.5 and 5.6.

Definition 5.2 In order to describe the homotopy fiber the map (9) of Definition 3.9, we need a cofibrant replacement of $\mathcal{A} c t_{>0}$ as a colored operad. Since $\mathcal{A c t} t_{>0}$ is cofibrant as an $\{o ; c\}$-sequence, we know from [3] that the Boardman-Vogt resolution of $\mathcal{A c t}_{>0}$, denoted by $B V\left(\mathcal{A} t_{>0}\right)$ or just $\mathcal{W A}$ in our case, is the object we are looking for. We recall the construction here.

- Let tree ${ }_{n}^{o}$ be the subset of $\{o ; c\}$-trees consisting of trees $(t, f)$ with $n$ leaves, where $f$ is an $\{o ; c\}$-labeling of $t$ with the trunk labeled by $o$ and satisfying, for all $v \in V(t)$,

$$
\begin{aligned}
& f\left(e_{0}(v)\right)=c \quad \Longrightarrow \quad f\left(e_{i}(v)\right)=c \text { for all } i \in\{1, \ldots,|v|\} \\
& f\left(e_{0}(v)\right)=o \quad \Longrightarrow \quad|v|>0, f\left(e_{|v|}(v)\right)=o \text { and } f\left(e_{i}(v)\right)=c \text { for } i \in\{1, \ldots,|v|-1\}
\end{aligned}
$$

- The operad $\mathcal{W A}$ is the $\{o ; c\}$-sequence given by

$\mathcal{W A}(n ; c):=$

$$
\coprod_{t \in\{c\} \text {-tree }} \prod_{v \in V(t)} \mathcal{A} c t_{>0}\left(f\left(e_{1}(v), \ldots, f\left(e_{|v|}(v)\right) ; f\left(e_{0}(v)\right)\right) \times \prod_{e \in E^{\text {int }}(t)}[0,1] / \sim,\right.
$$

$\mathcal{W A}(n ; o):=$

$$
\coprod_{t \in \operatorname{tree}_{n}^{o}} \prod_{v \in V(t)} \mathcal{A c t}_{>0}\left(f\left(e_{1}(v), \ldots, f\left(e_{|v|}(v)\right) ; f\left(e_{0}(v)\right)\right) \times \prod_{e \in E^{\text {int }}(t)}[0,1] / \sim\right.
$$


and the empty set otherwise. The equivalence relation $\sim$ is generated by contracting the inner edges indexed by 0 , using the operadic structure of $\mathcal{A c t}_{>0}$ and the following relation:

$$
\left.\oint_{t_{1}} \sim \mid \sim\right\}^{t_{1}} ; \quad \oint_{t_{2}}^{t_{1}} \sim \mid \max \left(t_{1} ; t_{2}\right)
$$

A point in $\mathcal{W A}(n ; o)$ will be denoted by $\left[T ;\left\{t_{e}\right\}\right]$, where $T$ is an element in tree ${ }_{n}^{o}$ such that each vertex has at least two inputs and $t_{e} \in[0,1]$ for each inner edge $e \in E^{\text {int }}(T)$. Similarly, a point in $\mathcal{W} \mathcal{A}(n ; c)$ is denoted by $\left[T ;\left\{t_{e}\right\}\right]$ with $T$ an element in $\{c\}$-tree. We will use the notation $v_{1}<v_{2}$ if $v_{1}$ and $v_{2}$ are two distinct, connected vertices such that $d\left(v_{1} ; r\right)<d\left(v_{2} ; r\right)$.

The operadic composition $\circ_{i}$ of two points $\left[T ;\left\{t_{e}\right\}\right]$ and $\left[T^{\prime} ;\left\{t_{e}^{\prime}\right\}\right]$ consists of grafting the tree $T^{\prime}$ to the $i^{\text {th }}$ leaf of $T$ and labeling the new inner edge by 1 .

- It is well known that the operad $\square:=\{\square(n)=\mathcal{W A}(n ; c)\}_{n>0}$ is a cofibrant replacement of $\mathrm{As}_{>0}$ as an operad. It is usually called the Stasheff operad.

The operad $\mathcal{W} \mathcal{A}$ has been introduced in [13] so as to recognize $A_{\infty}$-spaces and $A_{\infty}-$ maps. The next definition is a description, using the cofibrant replacement $\mathcal{W A}$, of the relative loop space $\Omega\left(\operatorname{Operad}^{h}\left(\operatorname{As}_{>0} ; O\right) ; \operatorname{Operad}_{\{o ; c\}}^{h}\left(\mathcal{A c t} t_{>0} ; X\right)\right)$.

Definition 5.3 Define $\Omega\left(\operatorname{Operad}\left(\triangle ; X_{c}\right)\right.$; $\left.\operatorname{Operad}_{\{o ; c\}}(\mathcal{W A} ; X)\right)$ as the space of maps given, for $n>0$, by

$$
g_{n ; c}: \mathcal{W A}(n ; c) \times[0,1] \rightarrow X(n ; c) \text { and } g_{n ; o}: \mathcal{W A}(n ; o) \times\{1\} \rightarrow X(n ; o),
$$

satisfying the relations

$$
\begin{array}{r}
g_{n ; c}\left(x \circ_{i} y ; t\right)=g_{l+1 ; c}(x ; t) \circ_{i} g_{n-l ; c}(y ; t) \\
\text { for } x \in \mathcal{W} \mathcal{A}(l+1 ; c), y \in \mathcal{W A}(n-l ; c) \text { and } i \in\{1, \ldots, l+1\}, \\
g_{n ; o}\left(x \circ_{i} y ; 1\right)=g_{l+1 ; o}(x ; 1) \circ_{i} g_{n-l ; c}(y ; 1) \\
\quad \text { for } x \in \mathcal{W A}(l+1 ; o), y \in \mathcal{W A}(n-l ; c) \text { and } i \in\{1, \ldots, l\}, \\
g_{n ; o}\left(x \circ_{l+1} y ; 1\right)=g_{l+1 ; o}(x ; 1) \circ_{l+1} g_{n-l ; o}(y ; 1) \\
\text { for } x \in \mathcal{W A}(l+1 ; o) \text { and } y \in \mathcal{W A}(n-l ; o),
\end{array}
$$

and the boundary condition $g_{n ; c}(x ; 0)=\eta\left(*_{n ; c}\right)$ for $x \in \mathcal{W A}(n ; c)$.

Notation 5.4 Let $\alpha$ : As $>0 \rightarrow O$ be a map of operads, $\beta: O \rightarrow B$ be a map of $O$-bimodules and $\eta: \mathcal{A} c t_{>0} \rightarrow X$ the corresponding map of $\{o ; c\}$-operads. The $\{o ; c\}$-sequence $X^{*}$ given by

$$
X^{*}(n ; c)=\eta\left(*_{n ; c}\right) \quad \text { for } n \geq 0, \quad X^{*}(n ; o)=B(n-1) \quad \text { for } n>0,
$$


and the empty set otherwise, inherits from $X$ an $\{o ; c\}$-operadic structure endowed with a map $\eta: \mathcal{A} c t_{>0} \rightarrow X^{*}$.

Proposition 5.5 Under assumption (13), the space $\operatorname{Bimod}_{\mathrm{As}_{>0}}^{h}\left(\mathrm{As}_{>0} ; B\right)$ is weakly equivalent to $\operatorname{Operad}_{\{o ; c\}}^{h}\left(\mathcal{A c t}>0 ; X^{*}\right)$.

Proof By assumption, $B$ is an $\mathrm{As}_{>0}$-bimodule. The first step of the proof consists of building a cofibrant replacement $\widetilde{\triangle}$ of $A s_{>0}$ as an $A s_{>0}$-bimodule such that there exists a map $\xi: \operatorname{Operad}_{\{o ; c\}}\left(\mathcal{W} \mathcal{A} ; X^{*}\right) \rightarrow \operatorname{Bimod}_{\mathrm{As}_{>0}}(\widetilde{\Delta} ; B)$. Let us recall that a point $g \in \operatorname{Operad}\left(\mathcal{W} \mathcal{A} ; X^{*}\right)$ is described by

$$
\begin{array}{ll}
g_{n ; c}: \mathcal{W} \mathcal{A}(n ; c) \rightarrow X^{*}(n ; c), \quad x \mapsto \eta\left(*_{n ; c}\right) & \text { for } n>0, \\
g_{n ; o}: \mathcal{W} \mathcal{A}(n ; o) \rightarrow X^{*}(n ; o) & \text { for } n>0,
\end{array}
$$

satisfying, notably for $x \in \mathcal{W} \mathcal{A}(l+1 ; o), y \in \mathcal{W} \mathcal{A}(n-l ; c)$ and $1 \leq i \leq l$, the relation

$$
g_{n ; o}\left(x \circ_{i} y\right)=g_{l+1 ; o}(x) \circ_{i} g_{n-l ; c}(y)=g_{l+1 ; o}(x) \circ_{i} \eta\left(*_{n-l ; c}\right) .
$$

Define $\approx$ to be the equivalence relation on $\mathcal{W} \mathcal{A}(n ; o)$ generated by $\left[T ;\left\{t_{e}\right\}\right] \approx\left[T ;\left\{l_{e}\right\}\right] \Longleftrightarrow \begin{cases}t_{e}=l_{e} & \forall e \in E^{\text {int }}(T) \text { with } f(e)=o, \text { and } \\ t_{e}=l_{e} & \text { if } \nexists e_{1}<e \text { such that } t_{e_{1}}=l_{e_{1}}=1 \text { and } f\left(e_{1}\right)=c .\end{cases}$

We will denote by $\widetilde{\Delta}$ the sequence $\{\widetilde{\Delta}(n)=\mathcal{W} \mathcal{A}(n+1 ; o) / \approx\}_{n>0}$. By convention, $\widetilde{\triangle}(0)$ is the empty set.

Due to relation (14), the map $g$ induces a sequence map $\widetilde{g}:=\left\{\widetilde{g}_{n}: \widetilde{\Delta}(n) \rightarrow B(n)\right\}_{n>0}$. Let us prove that $\widetilde{\Delta}$ is a cofibrant replacement of $A s_{>0}$ as an $A s_{>0}$-bimodule. The bimodule structure is given, for $1 \leq i \leq n$, by

(i) $-\circ^{i} *_{2}: \widetilde{\Delta}(n) \rightarrow \widetilde{\Delta}(n+1), \quad\left[T ;\left\{t_{e}\right\}\right] \mapsto\left[T ;\left\{t_{e}\right\}\right] \circ_{i} \delta_{2 ; c}$,

(ii) $*_{2}(-;-): \widetilde{\Delta}(n) \times \widetilde{\square}(m) \rightarrow \widetilde{\Delta}(n+m), \quad\left(\left[T_{1} ;\left\{t_{e}\right\}\right] ;\left[T_{2} ;\left\{l_{e}\right\}\right]\right)$

$$
\mapsto\left[T_{1} ;\left\{t_{e}\right\}\right] \circ_{n+1}\left[T_{2} ;\left\{l_{e}\right\}\right],
$$

where $\delta_{n ; c}$ is the $n$-corolla in $\{c\}$-trees and $\delta_{n ; o}$ is the $n$-corolla in tree ${ }_{n}^{o}$. This structure satisfies the bimodule axioms over $\mathrm{As}_{>0}$, and it makes $\tilde{f}$ into an $\mathrm{As}_{>0}-$ bimodule map. Furthermore $\widetilde{\triangle}$ is a cofibrant replacement.

Cofibrant Let $\widetilde{\square}_{n}$ be the $\mathrm{As}_{>0}$-bimodule generated by $\{\widetilde{\Delta}(i)\}_{i=1}^{n}$ for $n>0$. By convention, $\widetilde{\triangle}_{0}$ is the $\mathrm{As}_{>0}$-bimodule $B_{\mathrm{As}_{>0}}(\varnothing)$. Let us notice that the map $\mathcal{W} \mathcal{A}(n+1 ; o) \rightarrow \widetilde{\Delta}(n)$ preserves the boundary, and a point in $\partial \mathcal{W} \mathcal{A}(n+1 ; o)$, by definition, has the form $\left[T ;\left\{t_{e}\right\}\right]$ such that there exists $e_{1} \in E^{\text {int }}(T)$ with $t_{e_{1}}=1$ and either $f\left(e_{1}\right)=o$ or $f\left(e_{1}\right)=c$. If $f\left(e_{1}\right)=o$, then $\left[T ;\left\{t_{e}\right\}\right]$ has a decomposition 
$\left[T_{1} ;\left\{t_{e}^{1}\right\}\right] \circ_{\left|T_{1}\right|}\left[T_{2} ;\left\{t_{e}^{2}\right\}\right]$. The image lies in $\widetilde{\square}_{n-1}$ by axiom (ii). If $f\left(e_{1}\right)=c$, then $\left[T ;\left\{t_{e}\right\}\right]$ has a similar decomposition $\left[T_{1} ;\left\{t_{e}^{1}\right\}\right] \circ_{i}\left[T_{2} ;\left\{t_{e}^{2}\right\}\right]$ with $i<\left|T_{1}\right|$ and $\left|T_{2}\right|>1$, and we have the identification

$$
\left[T ;\left\{t_{e}\right\}\right]=\left[\left(T_{1} ;\left\{t_{e}^{1}\right\}\right) \circ_{i}\left(T_{2} ;\left\{t_{e}^{2}\right\}\right)\right]=\left[\left(T_{1} ;\left\{t_{e}^{1}\right\}\right) \circ_{i} \delta_{\left|T_{2}\right| ; c}\right]=\left[\left(T_{1} ;\left\{t_{e}^{1}\right\}\right) \circ_{i} \delta_{\left|T_{2}\right|-1 ; c}\right] \circ^{i} *_{2} .
$$

Hence $\left[T ;\left\{t_{e}\right\}\right]$ lies in $\widetilde{\square}_{n-1}$. Consequently, $\widetilde{\Delta}_{n}$ is obtained from $\widetilde{\square}_{n-1}$ by the pushout diagram

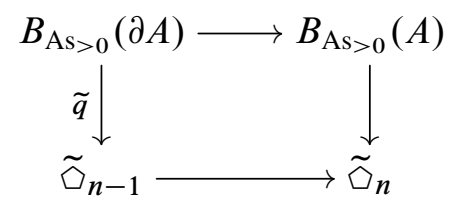

where $A$ is the sequence given by $A(n)=\mathcal{W} \mathcal{A}(n+1 ; o)$ and the empty set otherwise. The attaching map is the restriction of the quotient map $q: \mathcal{W A}(n+1 ; o) \rightarrow \widetilde{\square}(n)$ to the boundary. Furthermore, if $i \geq n$, then $\widetilde{\Delta}_{i}(n)=\widetilde{\Delta}(n)$, and the map $\partial A \rightarrow A$ is a cofibration. So $\lim _{i} \widetilde{\Delta}_{i}=\widetilde{\Delta}$, and thus $\widetilde{\Delta}$ is cofibrant. Like in the proof of Proposition 4.4, these sequences of pushout diagram imply that, for each $n$, the space $\widetilde{\square}(n)$ is a CW-complex.

Contractible The map $q: \mathcal{W A}(n+1 ; o) \rightarrow \widetilde{\square}(n)$ is a continuous map between compact CW-complexes. Since the fiber of $q$ over a point is homeomorphic to a product of polytopes that is contractible, the map $q$ is a weak equivalence; see [18, Main Theorem]. Hence $\widetilde{\square}(n)$ is contractible for $n>0$.

Since $\widetilde{\Delta}$ is a cofibrant replacement of $\mathrm{As}_{>0}$ as a bimodule over itself, the space $\operatorname{Bimod}_{\mathrm{As}>0}^{h}\left(\mathrm{As}_{>0} ; B\right)$ is weakly equivalent to $\operatorname{Bimod}_{\mathrm{As}_{>0}}(\widetilde{\Delta} ; B)$, and the assignment $\xi(g)=\widetilde{g}$ defines a map

$$
\xi: \operatorname{Operad}_{\{o ; c\}}\left(\mathcal{W} \mathcal{A} ; X^{*}\right) \rightarrow \operatorname{Bimod}_{\mathrm{As}>0}(\widetilde{\Delta} ; B), \quad g \mapsto \tilde{g} .
$$

In order to prove that $\xi$ is a weak equivalence, we introduce two towers of fibrations. Define $A_{k}^{\prime}$ and $B_{k}^{\prime}$ to be the subspaces

$$
A_{k}^{\prime} \subset \underbrace{\prod_{i=1}^{k+1} \operatorname{Top}\left(\mathcal{W} \mathcal{A}(i ; c) ; X^{*}(i ; c)\right)}_{\text {reduced to a point }} \times \prod_{i=1}^{k+1} \operatorname{Top}\left(\mathcal{W A}(i ; o) ; X^{*}(i ; o)\right)
$$

and

$$
B_{k}^{\prime} \subset \prod_{i=1}^{k} \operatorname{Top}(\widetilde{\square}(i) ; B(i)),
$$


with $A_{k}^{\prime}$ satisfying the operadic relations and $B_{k}^{\prime}$ the $\mathrm{As}_{>0}$-bimodule relations for $k>0$. In other words, $A_{k}^{\prime}$ and $B_{k}^{\prime}$ are respectively the space $\operatorname{Operad}\left(\mathcal{W} \mathcal{A}_{k+1} ; X^{*}\right)$ and $\operatorname{Bimod}_{\mathrm{As}>0}\left(\widetilde{\square}_{k} ; B\right)$, where $\mathcal{W} \mathcal{A}_{k+1}$ is the suboperad of $\mathcal{W} \mathcal{A}$ generated by $\{\mathcal{W} \mathcal{A}(i ; c) \mid$ $1 \leq i \leq k+1\}$ and $\{\mathcal{W} \mathcal{A}(i ; o) \mid 1 \leq i \leq k+1\}$. Since $\mathcal{W} \mathcal{A}(1 ; c)$ and $\mathcal{W} \mathcal{A}(1 ; o)$ are reduced to the unit, the factors $\operatorname{Top}\left(\mathcal{W} \mathcal{A}(1 ; c) ; X^{*}(1 ; c)\right)$ and $\operatorname{Top}\left(\mathcal{W} \mathcal{A}(1 ; o) ; X^{*}(1 ; o)\right)$ are one point spaces and can be ignored. So we consider the two towers

$$
\begin{aligned}
& A_{1}^{\prime} \longleftarrow A_{2}^{\prime} \longleftarrow \cdots \longleftarrow A_{k}^{\prime} \longleftarrow A_{k+1}^{\prime} \longleftarrow \cdots \\
& B_{1}^{\prime} \longleftarrow B_{2}^{\prime} \longleftarrow \cdots \longleftarrow B_{k}^{\prime} \longleftarrow B_{k+1}^{\prime} \longleftarrow \cdots
\end{aligned}
$$

such that

$$
\begin{aligned}
& A_{\infty}^{\prime}=\lim _{k} A_{k}^{\prime} \simeq \underset{k}{\operatorname{holim}} A_{k}^{\prime} \simeq \operatorname{Operad}_{\{o ; c\}}\left(\mathcal{W} \mathcal{A} ; X^{*}\right), \\
& B_{\infty}^{\prime}=\lim _{k} B_{k}^{\prime} \simeq \operatorname{holim}_{k} B_{k}^{\prime} \simeq \operatorname{Bimod}_{\mathrm{As}>0}(\widetilde{\square} ; B) .
\end{aligned}
$$

By restriction, the map $\xi$ induces a map between the two towers

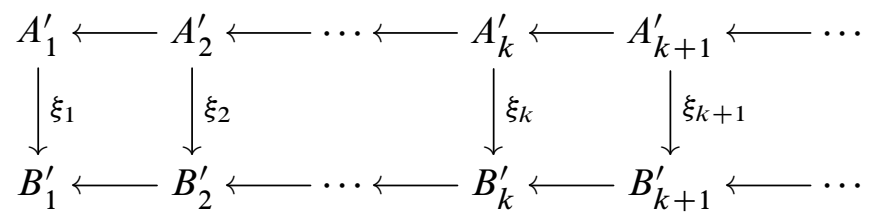

with $\xi=\lim _{k} \xi_{k}=\operatorname{holim}_{k} \xi_{k}$. We will prove that $\xi$ is a weak equivalence if each $\xi_{k}$ is a weak equivalence by induction on $k$. First, note that $\xi_{1}$ coincides with the identity. It is a weak equivalence. Next, assume that $\xi_{k-1}$ is a weak equivalence. We consider the following diagram, where $g$ is a point in $A_{k-1}^{\prime}, F_{A^{\prime}}$ is the fiber over $g$ and $F_{B^{\prime}}$ the fiber over $\xi_{k-1}(g)$ :

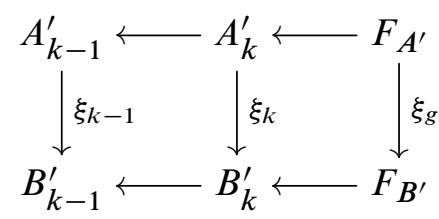

Since the two left horizontal arrows are fibrations, the map $\xi_{k}$ is a weak equivalence if the induced map $\xi_{g}$ is a weak equivalence.

From Lemma 3.8, the space $\operatorname{Top}^{g_{k+1 ; o}}((\mathcal{W} \mathcal{A}(k+1 ; o) ; \partial \mathcal{W} \mathcal{A}(k+1 ; o)) ; B(k))$ is homeomorphic to the fiber $F_{A^{\prime}}$. Similarly, $\widetilde{\square}_{k}$ is obtained from $\widetilde{\square}_{k-1}$ by the pushout diagram (15). So the space Top $\xi_{k-1}(g)_{k} \circ q((\mathcal{W A}(k+1 ; o) ; \partial \mathcal{W} \mathcal{A}(k+1 ; o)) ; B(k))$ is 
homeomorphic to the fiber $F_{B^{\prime}}$, and we have the following commutative square:

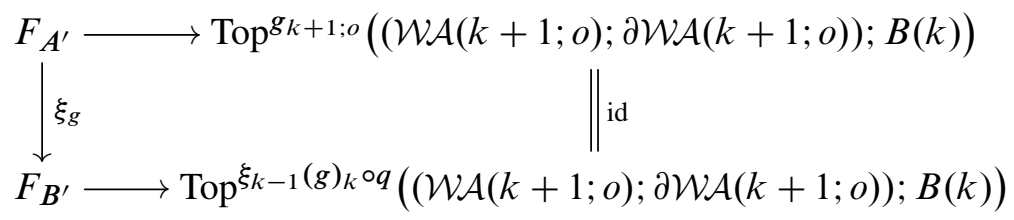

Consequently, $\xi_{k}$ is a weak equivalence.

Proposition 5.6 Under assumption (13), the space Operad $h_{\{o ; c\}}^{h}\left(\mathcal{A c t}_{>0} ; X^{*}\right)$ is weakly equivalent to the relative loop space $\Omega\left(\operatorname{Operad}^{h}\left(\operatorname{As}_{>0} ; X_{c}\right) ; \operatorname{Operad}_{\{o ; c\}}^{h}\left(\mathcal{A c t}_{>0} ; X\right)\right)$.

Proof Using the following inclusion, we can consider $\operatorname{Operad}_{\{o ; c\}}\left(\mathcal{W} \mathcal{A} ; X^{*}\right)$ as a subspace of $\Omega\left(\operatorname{Operad}\left(\triangle ; X_{c}\right) ; \operatorname{Operad}_{\{o ; c\}}(\mathcal{W} \mathcal{A} ; X)\right)$.

$$
\begin{aligned}
i: \operatorname{Operad}_{\{o ; c\}}\left(\mathcal{W} \mathcal{A} ; X^{*}\right) & \rightarrow \Omega\left(\operatorname{Operad}\left(\bullet ; X_{c}\right) ; \operatorname{Operad}_{\{o ; c\}}(\mathcal{W} \mathcal{A} ; X)\right) \\
g & \mapsto \begin{cases}\widetilde{g}_{n ; c}: \mathcal{W} \mathcal{A}(n ; c) \times[0,1] \rightarrow X(n ; c), & (x ; t) \mapsto \eta\left(*_{n ; c}\right) \\
\widetilde{g}_{n ; o}: \mathcal{W} \mathcal{A}(n ; o) \times\{1\} \rightarrow X(n ; o), & (x ; 1) \mapsto g_{n ; o}(x)\end{cases}
\end{aligned}
$$

In order to show that $i$ is a weak equivalence, we introduce two towers of fibrations. One of them is the tower $A_{k}^{\prime}$ of Proposition 5.5. The second one is defined by

$$
C_{k}^{\prime} \subset \prod_{i=1}^{k+1} \operatorname{Top}(\mathcal{W A}(i ; c) \times[0,1] ; X(i ; c)) \times \prod_{i=1}^{k+1} \operatorname{Top}(\mathcal{W} \mathcal{A}(i ; o) ; X(i ; o)),
$$

satisfying the relations of Definition 5.3. Since $\mathcal{W} \mathcal{A}(1 ; c)$ and $\mathcal{W} \mathcal{A}(1 ; o)$ are reduced to the unit, the factors $\operatorname{Top}(\mathcal{W} \mathcal{A}(1 ; c) \times[0,1] ; X(1 ; c))$ and $\operatorname{Top}(\mathcal{W} \mathcal{A}(1 ; o) ; X(1 ; o))$ are the one point space and can be ignored. The restriction to the space $A_{k}^{\prime}$ of the inclusion $i$ induces a map between the two towers as follows:

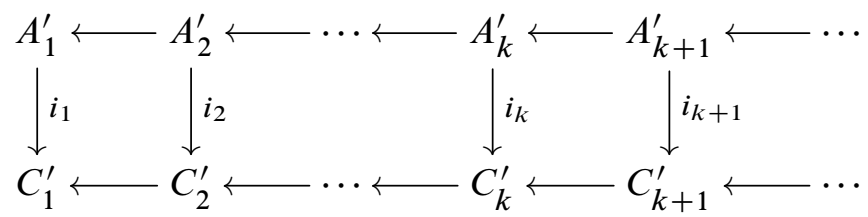

Since the space $\Omega\left(\operatorname{Operad}\left(\triangle ; X_{c}\right)\right.$; $\left.\operatorname{Operad}_{\{o ; c\}}(\mathcal{W} \mathcal{A} ; X)\right)$ is weakly equivalent to the limit of $C_{k}^{\prime}$, the map $i$ is a weak equivalence if each $i_{k}$ is a weak equivalence. We will prove this result by induction on $k$.

If $k=1$, a point in $C_{1}^{\prime}$ is a pair $\left(g_{2 ; c} ; g_{2 ; o}\right)$, whereas the points in the image of $i_{1}$ coincide with the pairs satisfying

$$
g_{2 ; c}: \mathcal{W A}(2 ; c) \times[0,1] \rightarrow X(2 ; c), \quad(x ; t) \mapsto \eta\left(*_{2 ; c}\right) .
$$


Since $g_{2 ; c}(x ; 0)=\eta\left(*_{1 ; c}\right)$ for any pair in $C_{1}^{\prime}$, the inclusion $i_{1}$ induces the following deformation retract:

$H: C_{1}^{\prime} \times[0,1] \rightarrow C_{1}^{\prime}, \quad y=\left(\left(g_{2 ; c} ; g_{2 ; o}\right) ; t_{1}\right) \mapsto\left\{\begin{array}{l}H(y)_{2 ; c}\left(\delta_{2 ; c} ; t\right)=g_{2 ; c}\left(\delta_{2 ; c} ; t\left(1-t_{1}\right)\right), \\ H(y)_{2 ; o}\left(\delta_{2 ; o} ; 1\right)=g_{2 ; o}\left(\delta_{2 ; o} ; 1\right) .\end{array}\right.$

From now on we assume that $i_{k-1}$ is a weak equivalence for $k \geq 2$. We consider the following diagram, where $g$ is a point in $A_{k-1}^{\prime}, F_{A^{\prime}}$ the fiber over $g$ and $F_{C^{\prime}}$ the fiber over $i_{k-1}(g)$ :

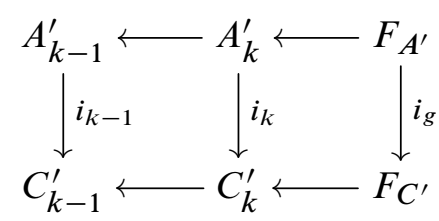

Since the two left horizontal arrows are fibrations, the map $i_{k}$ is a weak equivalence if the induced map $i_{g}$ is a weak equivalence.

A point in the fiber $F_{C^{\prime}}$ is defined by a pair $\left(g_{k+1 ; c} ; g_{k+1 ; o}\right)$ satisfying the relations of Definition 5.3. Since the pair is in the fiber over $i_{k-1}(g)$, the map $g_{k+1 ; c}$ sends all the faces of $\mathcal{W} \mathcal{A}(k+1 ; c) \times[0,1]$ to $\eta\left(*_{k+1 ; c}\right)$, except for the face $\mathcal{W} \mathcal{A}(k+1 ; c) \times\{1\}$.

On the other hand, the points in the image of $i_{g}$ coincide with the pairs $\left(g_{k+1 ; c} ; g_{k+1 ; o}\right)$ such that

$$
g_{k+1 ; c}: \mathcal{W} \mathcal{A}(k+1 ; c) \times[0,1] \rightarrow X(k+1 ; c), \quad(x ; t) \mapsto \eta\left(*_{k+1 ; c}\right) .
$$

In order to prove that $i_{g}$ induces a deformation retract, we consider a lift $H^{\prime}$ in the following diagram:

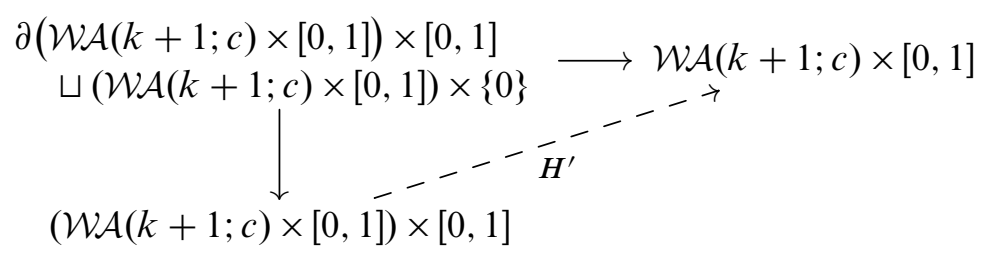

Here, the horizontal arrow is the inclusion on the factor $(\mathcal{W A}(k+1 ; c) \times[0,1]) \times\{0\}$ and sends a point $\left(\left(x ; t_{1}\right) ; t_{2}\right) \in \partial(\mathcal{W} \mathcal{A}(k+1 ; c) \times[0,1]) \times[0,1]$ to $\left(x ;\left(1-t_{2}\right) t_{1}\right)$. Such a lift exists since the vertical arrow is an acyclic cofibration and the space $\mathcal{W} \mathcal{A}(k+1 ; c) \times[0,1]$ 
is fibrant. The homotopy $H^{\prime}$ is illustrated by the following picture:

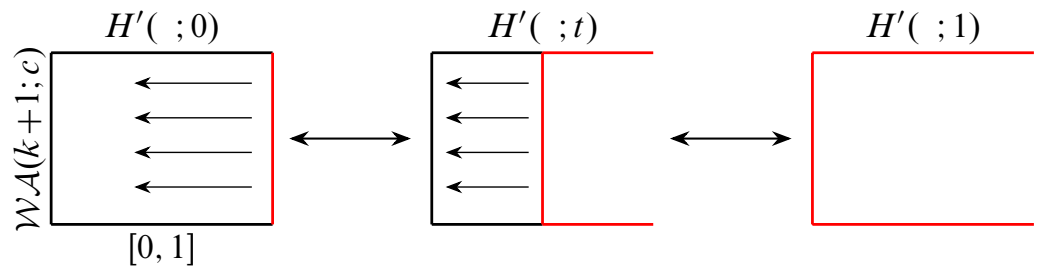

In other words, the points in the image of $i_{g}$ coincide with the pairs such that

$$
g_{k+1 ; c}(x ; t)=g_{k+1 ; c}\left(H^{\prime}((x ; t) ; 1)\right)=\eta\left(*_{k+1 ; c}\right)
$$

for $x \in \mathcal{W} \mathcal{A}(k+1 ; c)$ and $t \in[0,1]$. Finally, the deformation retract

$$
H_{2}: F_{C^{\prime}} \times[0,1] \rightarrow F_{C^{\prime}}
$$

sends a point $y=\left(\left(g_{k+1 ; c} ; g_{k+1 ; o}\right) ; t_{1}\right)$ to

$$
\begin{cases}H_{2}(y)_{k+1 ; c}(x ; t)=g_{k+1 ; c}\left(H^{\prime}\left((x ; t) ; t_{1}\right)\right) & \text { for } x \in \mathcal{W} \mathcal{A}(k ; c) \text { and } t \in[0,1], \\ H_{2}(y)_{k+1 ; o}(x ; 1)=g_{k+1 ; o}(x ; 1) & \text { for } x \in \mathcal{W} \mathcal{A}(k+1 ; o) .\end{cases}
$$

Consequently, we have that $\Omega\left(\operatorname{Operad}\left(\triangle ; X_{c}\right) ; \operatorname{Operad}_{\{o ; c\}}(\mathcal{W} \mathcal{A} ; X)\right)$ is weakly equivalent to $\operatorname{Operad}_{\{o ; c\}}\left(\mathcal{W} \mathcal{A} ; X^{*}\right)$.

Corollary 5.7 Let $\alpha$ : As $\rightarrow O$ be a map of operads and $\beta$ : $O \rightarrow B$ be a map of $O$-bimodules. Under assumption (13), if $B(0) \simeq *$ and $O(0) \simeq O(1) \simeq *$, then the pair $(\operatorname{sTot}(O) ; \operatorname{sTot}(B))$ is weakly equivalent to the $\mathcal{S C}_{2}$-space

$$
\left(\Omega^{2} \operatorname{Operad}^{h}\left(\operatorname{As}_{>0} ; O\right) ; \Omega^{2}\left(\operatorname{Operad}^{h}\left(\operatorname{As}_{>0} ; X_{c}\right) ; \operatorname{Operad}_{\{o ; c\}}^{h}\left(\mathcal{A c t}_{>0} ; X\right)\right)\right),
$$

where $X$ is the operad given by relations (12).

\section{Double relative delooping: general case}

In this section, $O$ is an $\{o ; c\}$-operad endowed with a map of operads $\eta: \mathcal{A} c t \rightarrow O$ that makes $O$ into an $\mathcal{A} c t_{>0}$-bimodule under $\mathcal{A} c t$. We denote by $\left(O_{c} ; O_{o}\right)$ the pair of semicosimplicial spaces associated to $O$; see Proposition 1.13. In Section 4, we proved that the pair $\left(\operatorname{sTot}\left(O_{c}\right) ; \operatorname{sTot}\left(O_{o}\right)\right)$ is weakly equivalent to

$\left(\Omega \operatorname{Bimod}_{\mathrm{As}>0}^{h}\left(\mathrm{As}_{>0} ; O_{c}\right) ; \Omega\left(\operatorname{Bimod}_{\mathrm{As}_{>0}}^{h}\left(\mathrm{As}_{>0} ; O_{c}\right) ; \operatorname{Bimod}_{\mathcal{A} c t_{>0}}^{h}(\mathcal{A c t}>0 ; O)\right)\right)$, under the assumption $O(0 ; c) \simeq *$.

If we assume that $O(1 ; c) \simeq *$, then $\operatorname{Bimod}_{\mathrm{As}>0}^{h}\left(\mathrm{As}_{>0} ; O_{c}\right) \simeq \Omega \operatorname{Operad}^{h}\left(\mathrm{As}_{>0} ; O_{c}\right)$. Similarly, Marcy D Robertson shows in [16] that the derived space of bimodule maps 
is weakly equivalent to the loop space of the derived space of operadic maps. More precisely, in our context, we have the following proposition.

Proposition 6.1 Let $O$ be an action-operad with $O(1 ; c) \simeq O(1 ; o) \simeq *$. The space $\operatorname{Bimod}_{\mathcal{A} c t>0}^{h}\left(\mathcal{A c t} t_{>0} ; O\right)$ is weakly equivalent to $\Omega \operatorname{Operad}_{\{o ; c\}}^{h}\left(\mathcal{A c t}_{>0} ; O\right)$.

Sketch of proof The proof is the same as in [19, Theorem 7.2]. According to the notation of Turchin, $\mathcal{B} \square$ is a cofibrant replacement of $A s_{>0}$ in the model category Bimod $_{\mathrm{As}>0}$ such that there exists a map

$$
\xi_{c}: \Omega \operatorname{Operad}\left(\triangle ; O_{c}\right) \rightarrow \operatorname{Bimod}_{\mathrm{As}>0}\left(\mathcal{B} \sqcup ; O_{c}\right), \quad f \mapsto \xi_{c}^{f} .
$$

Using towers of fibrations as we do in Sections 4 and 5, Turchin proves that $\xi_{c}$ is a weak equivalence. The construction of the map $\xi_{c}$ is obtained from a polytope subdivision $\mathcal{B} \square(n)=\{\mathcal{B} \sqcup(T)\}_{T}$ indexed by $\{c\}$-trees with $n$ leaves. More precisely, for any $\{c\}$-tree $T$ with $n$ leaves, the space $\mathcal{B} \square(T)$ is the product of the following two spaces:

$$
\begin{aligned}
& \lambda_{\square}(T)=\prod_{v \in V(T)} \square(|v|), \\
& \chi_{\Delta}(T)=\left\{\left\{t_{v}\right\}_{v \in V(T)} \mid t_{v} \in[0,1] \text { and } t_{v_{1}} \leq t_{v_{2}} \text { if } v_{1}<v_{2}\right\} \subset[0,1]^{|V(T)|} .
\end{aligned}
$$

A point in $\mathcal{B} \sqcup(T)$ is denoted by $\left\{x_{v} ; t_{v}\right\}$ with $\left\{x_{v}\right\} \in \lambda_{\triangle}(T)$ and $\left\{t_{v}\right\} \in \chi_{\Delta}(T)$. For any $f \in \Omega \operatorname{Operad}\left(\triangle ; O_{c}\right)$, the map $\xi_{c}^{f}$ is defined on each polytope $\mathcal{B} \backsim(T)$ by induction on the number of vertices of $T$ using the operadic structure of $O_{c}$ :

$$
\begin{aligned}
\xi_{T ; c}^{f}: \mathcal{B} \sqcup(T) & \rightarrow O(n ; c) \\
\left\{x_{v} ; t_{v}\right\} & \mapsto f_{|r|}\left(x_{r} ; t_{r}\right)\left(\xi_{T_{1} ; c}^{f}\left(\left\{x^{1} ; t^{1}\right\}\right), \ldots, \xi_{T_{|r|} ; c}^{f}\left(\left\{x^{|r|} ; t^{|r|}\right\}\right)\right),
\end{aligned}
$$

with $T_{i}$ the subtree of $T$ whose trunk coincides with the $i^{\text {th }}$ input edge of the root of $T$.

In our case, a cofibrant replacement of $\mathcal{A c t}_{>0}$ in the model category $\operatorname{Bimod}_{\mathcal{A} t>0}$ is the $\{o ; c\}$-sequence

$$
\mathcal{B} \triangle_{\{o ; c\}}(n ; c)=\mathcal{B} \checkmark_{\{o ; c\}}(n ; o)=\mathcal{B} \sqcup(n) \quad \text { for } n>0,
$$

and the empty set otherwise, with the obvious $\mathcal{A} c t_{>0}$-bimodule structure. The space $\mathcal{B} \triangle_{\{o ; c\}}(n ; o)$ has a polytope subdivision $\left\{\mathcal{B} \triangle_{\{o ; c\}}(T)\right\}_{T}$ indexed by tree ${ }_{n}^{o}$. The space 
$\mathcal{B} \triangle_{\{o ; c\}}(T)$ is the product of the following two spaces:

$$
\begin{aligned}
& \lambda_{\triangle}(T)=\prod_{v \in V(T)} \mathcal{W A}\left(n ; f\left(e_{0}(v)\right)\right), \\
& \chi_{\triangle}(T)=\left\{\left\{t_{v}\right\}_{v \in V(T)} \mid t_{v} \in[0,1] \text { and } t_{v_{1}}<t_{v_{2}} \text { if } v_{1}<v_{2}\right\} \subset[0,1]^{|V(T)|} .
\end{aligned}
$$

A point in $\mathcal{B} \square_{\{o ; c\}}(T)$ is denoted by $\left\{x_{v} ; t_{v}\right\}$. For any $f \in \Omega \operatorname{Operad}(\mathcal{W A} ; O)$, the map $\xi^{f}$ is defined by $\xi_{n ; c}^{f}$ as before, and $\xi_{n ; o}^{f}$ by induction on the number of vertices of $T$ using the operadic structure of $O$ :

$$
\begin{aligned}
\xi_{T ; o}^{f}: \mathcal{B} \triangle_{\{o ; c\}}(T) & \rightarrow O(n ; o) \\
\left\{x_{v} ; t_{v}\right\} & \mapsto f_{|r| ; o}\left(x_{r} ; t_{r}\right)\left(\xi_{T_{1} ; c}^{f}\left(\left\{x^{1} ; t^{1}\right\}\right), \ldots, \xi_{T_{|r|} ; o}^{f}\left(\left\{x^{|r|} ; t^{|r|}\right\}\right)\right),
\end{aligned}
$$

with $T_{i}$ the subtree of $T$ whose trunk coincides with the $i^{\text {th }}$ input edge of the root of $T$. It defines a map from $\Omega \operatorname{Operad}_{\{o ; c\}}(\mathcal{W} \mathcal{A} ; O)$ to $\operatorname{Bimod}_{\mathcal{A c t}>0}(\mathcal{B} \sqcup\{o ; c\} ; O)$, which is a weak equivalence using the same arguments as Turchin in [19].

Theorem 6.2 Assume $O$ is an $\{o ; c\}$-operad such that $O(0 ; c) \simeq O(1 ; c) \simeq *$ and $O(1 ; o) \simeq *$. If $\eta: \mathcal{A} c t \rightarrow O$ is a map of $\{o ; c\}$-operads, the pair $\left(\operatorname{sTot}\left(O_{c}\right) ; \operatorname{sTot}\left(O_{o}\right)\right)$ is weakly equivalent to the $\mathcal{S C}_{2}$-space

$$
\left(\Omega^{2} \operatorname{Operad}^{h}\left(\operatorname{As}_{>0} ; O_{c}\right) ; \Omega^{2}\left(\operatorname{Operad}^{h}\left(\operatorname{As}_{>0} ; O_{c}\right) ; \operatorname{Operad}_{\{o ; c\}}^{h}\left(\mathcal{A c t}_{>0} ; O\right)\right)\right) .
$$

Proof By [19, Theorem 7.2], we know that the space $\operatorname{sTot}\left(O_{c}\right)$ is weakly equivalent to $\Omega^{2} \operatorname{Operad}\left(\triangle ; O_{c}\right)$. Proposition 6.1 implies that the projection of $\xi$ onto the closed part gives rise to the commutative diagram

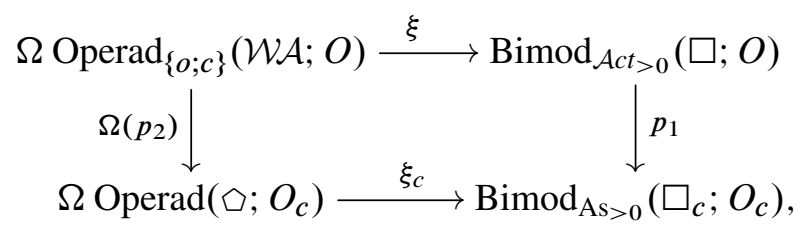

where $p_{1}$ and $p_{2}$ are respectively the maps (8) and (9). Since the homotopy fibers commute with the homotopy limits, we have the following:

$\Omega\left(\operatorname{Bimod}_{\mathrm{As}_{>0}}\left(\square_{c} ; O_{c}\right) ; \operatorname{Bimod}_{\mathcal{A} c t_{>0}}(\square ; O)\right)$

$$
\begin{aligned}
& \simeq \operatorname{hofib}\left(\operatorname{Bimod}_{\mathcal{A} c t>0}(\square ; O) \stackrel{p_{1}}{\longrightarrow} \operatorname{Bimod}_{\mathrm{As}>0}\left(\square_{c} ; O_{c}\right)\right) \\
& \simeq \operatorname{hofib}\left(\Omega \operatorname{Operad}_{\{o ; c\}}(\mathcal{W} \mathcal{A} ; O) \stackrel{\Omega\left(p_{2}\right)}{\longrightarrow} \Omega \operatorname{Operad}\left(\triangle ; O_{c}\right)\right) \\
& \simeq \Omega \operatorname{hofib}\left(\operatorname{Operad}_{\{o ; c\}}(\mathcal{W} \mathcal{A} ; O) \stackrel{p_{2}}{\longrightarrow} \operatorname{Operad}\left(\triangle ; O_{c}\right)\right) \\
& \simeq \Omega^{2}\left(\operatorname{Operad}\left(\triangle ; O_{c}\right) ; \operatorname{Operad}_{\{o ; c\}}(\mathcal{W} \mathcal{A} ; O)\right) .
\end{aligned}
$$




\section{Acknowledgments}

I would like to thank my $\mathrm{PhD}$ advisor, Muriel Livernet, for her input on this project. This paper probably never would have been finished if it was not for her help. I thank also Victor Turchin for his interest in this project and fruitful conversations.

\section{References}

[1] G Arone, V Turchin, On the rational homology of high-dimensional analogues of spaces of long knots, Geom. Topol. 18 (2014) 1261-1322 MR3228453

[2] C Berger, I Moerdijk, Axiomatic homotopy theory for operads, Comment. Math. Helv. 78 (2003) 805-831 MR2016697

[3] C Berger, I Moerdijk, The Boardman-Vogt resolution of operads in monoidal model categories, Topology 45 (2006) 807-849 MR2248514

[4] C Berger, I Moerdijk, Resolution of coloured operads and rectification of homotopy algebras, from: "Categories in algebra, geometry and mathematical physics", (A Davydov, M Batanin, M Johnson, S Lack, A Neeman, editors), Contemp. Math. 431, Amer. Math. Soc. (2007) 31-58 MR2342815

[5] J M Boardman, R M Vogt, Homotopy invariant algebraic structures on topological spaces, Lecture Notes in Mathematics 347, Springer, Berlin (1973) MR0420609

[6] E Dror, W G Dwyer, A long homology localization tower, Comment. Math. Helv. 52 (1977) 185-210 MR0458417

[7] W Dwyer, K Hess, Long knots and maps between operads, Geom. Topol. 16 (2012) 919-955 MR2928985

[8] W G Dwyer, J Spaliński, Homotopy theories and model categories, from: "Handbook of algebraic topology", (I M James, editor), North-Holland, Amsterdam (1995) 73-126 MR1361887

[9] B Fresse, Modules over operads and functors, Lecture Notes in Mathematics 1967, Springer, Berlin (2009) MR2494775

[10] J E Harper, Homotopy theory of modules over operads and non- $\Sigma$ operads in monoidal model categories, J. Pure Appl. Algebra 214 (2010) 1407-1434 MR2593672

[11] A Hatcher, Algebraic topology, Cambridge Univ. Press (2002) MR1867354

[12] PS Hirschhorn, Model categories and their localizations, Mathematical Surveys and Monographs 99, Amer. Math. Soc. (2003) MR1944041

[13] E Hoefel, M Livernet, J Stasheff, $A_{\infty}$-actions and recognition of relative loop spaces, preprint (2013) arXiv:1312.7155 
[14] M Hovey, Model categories, Mathematical Surveys and Monographs 63, Amer. Math. Soc. (1999) MR1650134

[15] J E McClure, J H Smith, Operads and cosimplicial objects: an introduction, from: "Axiomatic, enriched and motivic homotopy theory", (J P C Greenlees, editor), NATO Sci. Ser. II Math. Phys. Chem. 131, Kluwer, Dordrecht (2004) 133-171 MR2061854

[16] M Robertson, Spaces of operad structures, preprint (2011) arXiv:1111.3904

[17] D P Sinha, Operads and knot spaces, J. Amer. Math. Soc. 19 (2006) 461-486 MR2188133

[18] S Smale, A Vietoris mapping theorem for homotopy, Proc. Amer. Math. Soc. 8 (1957) 604-610 MR0087106

[19] V Turchin, Delooping totalization of a multiplicative operad, J. Homotopy Relat. Struct. 9 (2014) 349-418 MR3258687

[20] R M Vogt, Cofibrant operads and universal $E_{\infty}$ operads, Topology Appl. 133 (2003) 69-87 MR1996461

[21] A A Voronov, The Swiss-cheese operad, from: "Homotopy invariant algebraic structures”, (J-P Meyer, J Morava, W S Wilson, editors), Contemp. Math. 239, Amer. Math. Soc. (1999) 365-373 MR1718089

LAGA, CNRS, UMR 7539, Université Paris 13,

99 avenue Jean-Batiste Clément, 93430 Villetaneuse, France

ducoulombier@math.univ-paris13.fr

Received: 30 November 2014 Revised: 6 July 2015 